\title{
ASYMPTOTIC METHOD FOR INTERFACIAL SOLITARY WAVES IN A COMPRESSIBLE FLUID
}

\author{
M. C. Shen and S. M. Sun
}

\begin{abstract}
We justify rigorously an asymptotic method developed for twodimensional solitary waves at the interface between two isothermal layers of a perfect gas under gravity bounded by two rigid horizontal planes. Assuming that the two isothermal layers are in thermal inversion, we prove that if the reciprocal of the Froude number is smaller than but near the larger one of two critical values, the solitary wave solution obtained by the formal asymptotic method is indeed an approximate solution to the exact equations, thus establishing the mathematical existence of a solitary wave in this context.
\end{abstract}

\section{Introduction}

In recent years, there has been growing interest in studying interfacial progressive waves in an incompressible fluid. These problems generate many interesting wave patterns and require new mathematical methods to find solutions. They have been investigated numerically in [7]-[9], [15], and the mathematical existence of the interfacial solitary waves has been proved in [1]-[3], [11], [12], among others. Discussion of solitary waves in continuously stratified fluids can be found in the book on stratified flow by Yih [16] and the references cited there. Numerical studies of atmospheric interfacial waves, which are of much meteorological interest, also have been reported recently in [4] and [5]. Indeed, solitary pressure jumps at the interface between two isothermal layers in thermal inversion have long been observed to have a high correlation with the formation of tornados [13]. However, at present, a rigorous asymptotic theory of interfacial solitary waves in a compressible fluid is lacking, although some formal results are available [10].

In this paper, we justify rigorously the formal asymptotic method developed for two-dimensional interfacial solitary waves in a perfect gas with two isothermal layers in thermal inversion. This physical configuration is of interest in meteorological applications. We hope that our work may stimulate further research on rigorous asymptotic methods for progressive waves in other configurations of a compressible fluid, which pose many challenging mathematical questions. Let the constant temperatures in the upper and lower layers be $T_{2}$ and $T_{1}$, respectively, and $T_{1}<T_{2}$. For simplicity, the upper and lower boundaries of the fluid domain are assumed to be horizontal and rigid. However, the same method used here also could deal with a free surface as the upper boundary without much difficulty. A coordinate system moving with a solitary wave at a constant speed $c$ is chosen so that the fluid motion is steady. We define $\lambda=g h_{1}^{*} / c^{2}=\lambda_{0}+\epsilon \lambda_{1}$ as the reciprocal of the Froude number where $g$ is the constant gravitational acceleration, $h_{1}^{*}$ is the equilibrium height of the lower layer, and

Received September 8, 1995, revised November 22, 1995

1991 Mathematics Subject Classification. Primary: 76B25; secondary: 41A60.

Key words and phrases: asymptotic method, solitary waves, compressible fluid, isothermal layers, contraction mapping theorem. 
$\epsilon$ is a small positive parameter. Two critical values $\lambda_{ \pm}$of $\lambda_{0}$ have been found where $\lambda_{+}>\lambda_{-}>0$. For $\lambda$ near but less than $\lambda_{+}$, our main result is the following:

There exists an asymptotic solution, which represents an interfacial solitary wave, of the equations governing the motion of two isothermal layers of a perfect gas separated by an interface, bounded below and above by a rigid boundary. The expression of the interface is given by

$$
\eta(x)=\epsilon \psi_{0}^{-} \psi_{0}^{+} S\left(\epsilon^{1 / 2} x\right)+O\left(\epsilon^{2}\right) .
$$

Here $z=\eta(x)$ is the equation of the interface, $-\psi_{0}^{-}$and $\psi_{0}^{+}$are the constant values of a stream function at the lower and upper boundaries, respectively,

$$
S\left(\epsilon^{1 / 2} x\right)=\left(3 \lambda_{1} A_{1} / 2 A_{2}\right) \operatorname{sech}^{2}\left(\left(-\epsilon \lambda_{1} A_{1} / A_{0}\right)^{1 / 2} x / 2\right),
$$

$A_{i}, i=1,2,3$ are constants, $A_{0}, A_{1}>0, A_{2}$ is assumed to be nonzero, and $O\left(\epsilon^{2}\right)$ is a term of order $\epsilon^{2}$. Therefore, the first approximation in an asymptotic expansion of the interface equation is a solitary wave of elevation if $A_{2}<0$ or a solitary wave of depression if $A_{2}>0$. In both cases, $\lambda_{1}$ must be negative.

We also note that for $A_{2}=0$, our method fails and a solitary wave as a solution of the so-called modified Korteweg-deVries equation could be derived by a refined asymptotic method. We omit that rather tedious derivation, but refer the reader to a discussion of a similar but simpler case for an incompressible fluid in [11].

The paper is organized as follows. The problem is formulated in Section 2 where the interface is transformed into a fixed horizontal line by using the stream function and the horizontal coordinate as independent variables, and the so-called streamline function is used as one of the dependent variables. The density of the compressible fluid is not a function of the stream function as in the incompressible case, so we have to deal with a system of equations governing the streamline function and the density. In Section 3, the approximate solution of a solitary wave is derived by a formal asymptotic expansion and two critical values $\lambda_{ \pm}$of $\lambda_{0}$ are obtained. For $\lambda$ near $\lambda_{-}$, the linear part of the governing equation for the lower layer in the $x, \psi$ plane becomes hyperbolic, and the method developed for $\lambda_{0}=\lambda_{+}$does not apply. We defer this case to a further study, and will consider only $\lambda_{0}=\lambda_{+}$in this paper. In Section 4, we reformulate the governing equations and boundary conditions as a system of nonhomogeneous equations with linear terms as the dominant part of the system. A solvability condition is derived for the nonhomogeneous system, which eventually yields an approximate solitary wave solution. Some Banach spaces for later use also are introduced. In Section 5, we derive a priori estimates for the ordinary differential equation associated with the solvability condition and also for the partial differential equation governing the gas motion. Furthermore, we show that an eigenvalue problem derived from the linearized equations possesses nonpositive eigenvalues only. Therefore, in the case considered here, the appearance of a solitary wave with ripples at infinity [12] is excluded. The final existence result is proved in Section 6 by means of the contraction mapping theorem.

\section{Formulation}

We consider a perfect gas consisting of two layers bounded by two rigid plane boundaries. At equilibrium the lower layer is at temperature $T_{1}$ with height $h_{1}^{*}$; the upper layer is at temperature $T_{2}$ with height $h_{2}^{*}$, separated from the lower layer by a contact interface; $p_{0}$ is the pressure at the interface, and the densities at the sides of the 
interface are $\rho_{0}^{+}, \rho_{0}^{-}$for the upper and lower layers, respectively. Note that $T_{1}<T_{2}$ implies $\rho_{0}^{-}>\rho_{0}^{+}$for a perfect gas. Assume that there is a wave of permanent type moving with a constant velocity $c$ on the interface. A coordinate system moving with the wave is chosen such that the $x^{*}$-axis coincides with the equilibrium interface and the $z^{*}$-axis passes through the crest or the trough of the wave and is positive upward (Fig.1). In reference to this coordinate system, the wave is stationary. At infinity, the

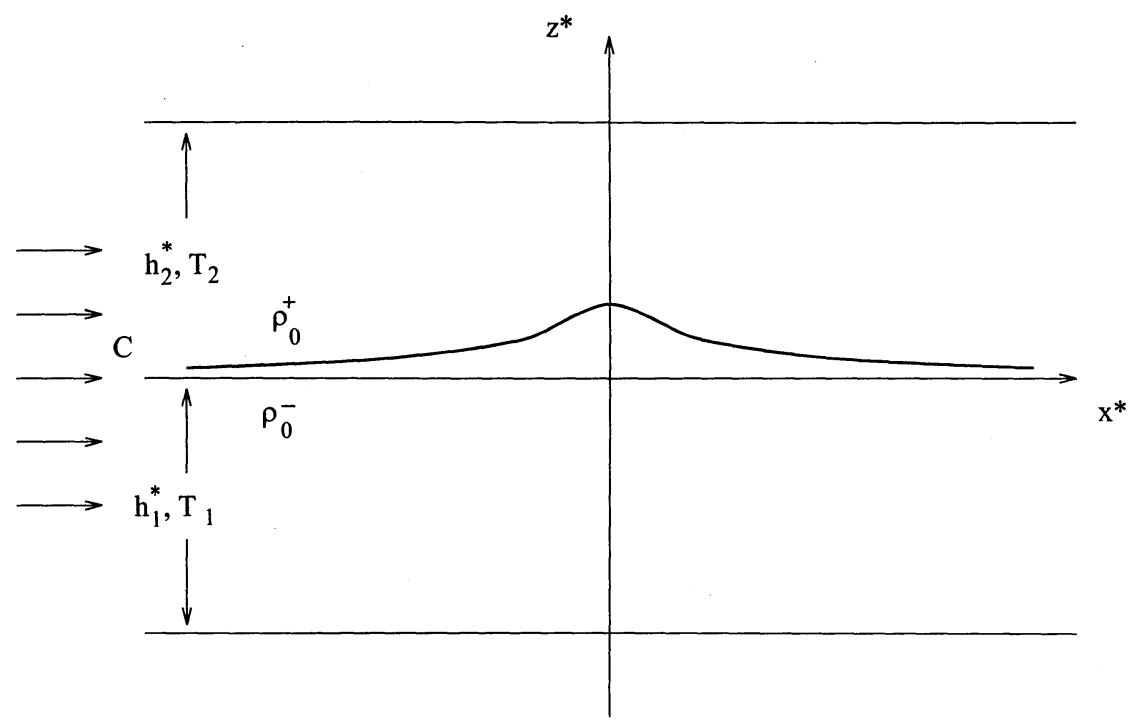

Figure 1. Configuration of the two-layer fluid.

gas is moving with constant speed $c$. The governing equations are

$$
\begin{aligned}
& \left(\rho^{*} u^{*}\right)_{x^{*}}+\left(\rho^{*} v^{*}\right)_{z^{*}}=0 \\
& u^{*} u_{x^{*}}^{*}+v^{*} u_{z^{*}}^{*}=-\left(\rho^{*}\right)^{-1} p_{x^{*}}^{*}, \\
& u^{*} v_{x^{*}}^{*}+v^{*} v_{z^{*}}^{*}=-g-\left(\rho^{*}\right)^{-1} p_{z^{*}}^{*}, \\
& p^{*}=C_{0}^{ \pm} \rho^{*}, \quad C_{0}^{+}=\frac{p_{0}}{\rho_{0}^{+}}, \quad C_{0}^{-}=\frac{p_{0}}{\rho_{0}^{-}} \\
& u_{z^{*}}^{*}-v_{x^{*}}^{*}=0
\end{aligned}
$$

where $\rho^{*}$ is the density, $\left(u^{*}, v^{*}\right)$ is the velocity vector, $p^{*}$ is the pressure, $\left(C_{0}^{+}\right)^{1 / 2}$ and $\left(C_{0}^{-}\right)^{1 / 2}$ are the sonic speeds of the upper and lower layers, respectively. The starred dependent variables are defined by

$$
f^{*}\left(x^{*}, z^{*}\right)= \begin{cases}\left(f^{*}\left(x^{*}, z^{*}\right)\right)^{+} & \text {for } \eta^{*}\left(x^{*}\right)<z^{*}<h_{2}^{*}, \\ \left(f^{*}\left(x^{*}, z^{*}\right)\right)^{-} & \text {for }-h_{1}^{*}<z^{*}<\eta^{*}\left(x^{*}\right),\end{cases}
$$

and $z^{*}=\eta^{*}\left(x^{*}\right)$ is the interface. At the rigid boundaries, $v^{*}=0$. At the interface, $u^{*} \eta_{x^{*}}^{*}-v^{*}=0$ and $\left(p^{*}\right)^{+}=\left(p^{*}\right)^{-}$. At infinity, $u^{*}=c, v^{*}=0$, and $\eta^{*}=0$ where $\left(p^{*}\right)^{+}=\left(p^{*}\right)^{-}=p_{0}$. It follows from (3) that

$$
\left(\rho^{*}\right)^{ \pm}=\rho_{0}^{ \pm} \exp \left(-\frac{g z^{*}}{C_{0}^{ \pm}}\right), \quad\left(p^{*}\right)^{ \pm}=C_{0}^{ \pm}\left(\rho^{*}\right)^{ \pm} .
$$

From (1), we can find a stream function $\psi^{*}$ such that

$$
\rho^{*} u^{*}=\psi_{z^{*}}^{*}, \quad \rho^{*} v^{*}=-\psi_{x^{*}}^{*} .
$$


At infinity,

$$
\left(\psi^{*}\right)^{ \pm}=\frac{c p_{0}}{g}\left(1-\exp \left(-\frac{g z^{*}}{C_{0}^{ \pm}}\right)\right)
$$

where $\psi^{*}=0$ at $z^{*}=0$ is assumed. Therefore, the values of $\psi^{*}$ at the upper and lower rigid boundaries are

$$
\psi_{s}^{+}=\frac{c p_{0}}{g}\left(1-\exp \left(-\frac{g h_{2}^{*}}{C_{0}^{+}}\right)\right), \quad \psi_{s}^{-}=\frac{c p_{0}}{g}\left(1-\exp \left(\frac{g h_{1}^{*}}{C_{0}^{-}}\right)\right) .
$$

By $(5), \psi^{*}$ satisfies

$$
\psi_{x^{*} x^{*}}^{*}+\psi_{z^{*} z^{*}}^{*}-\left(\rho^{*}\right)^{-1}\left(\psi_{x^{*}}^{*} \rho_{x^{*}}^{*}+\psi_{z^{*}}^{*} \rho_{z^{*}}^{*}\right)=0
$$

in the gas. Also, we have the following Bernoulli equation

$$
\left(\left(u^{*}\right)^{2}+\left(v^{*}\right)^{2}\right) / 2+g z^{*}+C_{0}^{ \pm} \ln \rho^{*}=\left(c^{2} / 2\right)+C_{0}^{ \pm} \ln \left(p_{0} / C_{0}^{ \pm}\right)
$$

from (2) and (3). Now we use $x^{*}, \psi^{*}$ as independent variables and the so-called streamline function $f^{*}\left(x^{*}, \psi^{*}\right)$ as a dependent variable where $\psi^{*}\left(x^{*}, f^{*}\right)=$ constant along a streamline, so that the domain of the streamline function is fixed. Thus,

$$
u^{*}=\frac{\psi_{z^{*}}^{*}}{\rho^{*}}=\frac{1}{\rho^{*} f_{\psi^{*}}^{*}}, \quad v^{*}=-\frac{\psi_{x^{*}}^{*}}{\rho^{*}}=-\frac{f_{x^{*}}^{*}}{\rho^{*} f_{\psi^{*}}^{*}} .
$$

The governing equations and boundary conditions in terms of $f^{*}$ become: in $-\psi_{s}^{-}<\psi^{*}<0,0<\psi^{*}<\psi_{s}^{+}$,

$$
\begin{aligned}
& f_{\psi^{*} \psi^{*}}^{*}\left(1+\left(f_{x^{*}}^{*}\right)^{2}\right)-2 f_{x^{*}}^{*} f_{\psi^{*}}^{*} f_{x^{*} \psi^{*}}^{*}+f_{x^{*} x^{*}}^{*}\left(f_{\psi^{*}}^{*}\right)^{2} \\
&+\left(1+\left(f_{x^{*}}^{*}\right)^{2}\right) f_{\psi^{*}}^{*} \frac{\rho_{\psi^{*}}^{*}}{\rho^{*}}-f_{x^{*}}^{*}\left(f_{\psi^{*}}^{*}\right)^{2} \frac{\rho_{x^{*}}^{*}}{\rho^{*}}=0 \\
& \frac{1+\left(f_{x^{*}}^{*}\right)^{2}}{2\left(\rho^{*} f_{\psi^{*}}^{*}\right)^{2}}+g f^{*}+C_{0}^{ \pm} \ln \rho^{*}=\left(c^{2} / 2\right)+C_{0}^{ \pm} \ln \left(p_{0} / C_{0}^{ \pm}\right)
\end{aligned}
$$

at $\psi^{*}=\psi_{s}^{+}>0$,

$$
\left(f^{*}\right)^{+}=h_{2}^{*}
$$

and at $\psi^{*}=0$,

$$
\begin{gathered}
\left(f^{*}\right)^{+}=\left(f^{*}\right)^{-} \\
\frac{1+\left(f_{x^{*}}^{*^{+}}\right)^{2}}{2 C_{0}^{+}\left(\rho^{*^{+}} f_{\psi^{*}}^{*^{+}}\right)^{2}}-\frac{1+\left(f_{x^{*}}^{*-}\right)^{2}}{2 C_{0}^{-}\left(\rho^{*^{-}} f_{\psi^{*}}^{*-}\right)^{2}}+g\left(\frac{f^{*^{+}}}{C_{0}^{+}}-\frac{f^{*^{-}}}{C_{0}^{-}}\right)=\left(c^{2} / 2\right)\left(\frac{1}{C_{0}^{+}}-\frac{1}{C_{0}^{-}}\right)
\end{gathered}
$$

and at $\psi^{*}=\psi_{s}^{-}<0$,

$$
\left(f^{*}\right)^{-}=-h_{1}^{*} .
$$

To nondimensionalize (12) to (17), we let

$$
\begin{aligned}
& f^{*}=h_{0} f, x^{*}=h_{0} x, \psi^{*}=\frac{c p_{0}}{g} \psi, \psi_{s}^{+}=\frac{c p_{0}}{g} \psi_{0}^{+}, \\
& \rho^{*}=\rho_{0} \rho=\left(\frac{p_{0}}{h_{0} g}\right) \rho, C_{0}^{ \pm}=g h_{0} C^{ \pm}, \psi_{s}^{-}=-\frac{c p_{0}}{g} \psi_{0}^{-}, \\
& h_{2}^{*}=h_{0} h^{+}, h_{1}^{*}=h_{0} h^{-}, \lambda=\left(g h_{0} / c^{2}\right),
\end{aligned}
$$


where $h_{0}$ is the length scale and can be chosen as $h_{1}^{*}$, and $\rho_{0}$ is the scale for density and can be set to $\rho_{0}^{-}$. Then (12) to (17) become: in $0<\psi<\psi_{0}^{+},-\psi_{0}^{-}<\psi<0$,

$$
\begin{aligned}
f_{\psi \psi}\left(1+f_{x}^{2}\right)-2 f_{x} f_{\psi} f_{x \psi} & +f_{x x} f_{\psi}^{2}+\left(1+f_{x}^{2}\right) f_{\psi} \sigma_{\psi}-f_{x} f_{\psi}^{2} \sigma_{x}=0, \\
\frac{e^{-2 \sigma}\left(1+f_{x}^{2}\right)}{2 f_{\psi}^{2}}+\lambda f+\lambda C^{ \pm} \sigma & =\frac{1}{2}-\lambda C^{ \pm} \ln C^{ \pm}
\end{aligned}
$$

at $\psi=\psi_{0}^{+}<1$

$$
f=h^{+}=-C^{+} \ln \left(1-\psi_{0}^{+}\right)
$$

at $\psi=0$,

$$
\begin{gathered}
f^{+}=f^{-} \\
\frac{1+\left(f_{x}^{+}\right)^{2}}{2 C^{+}\left(f_{\psi}^{+}\right)^{2}} e^{-2 \sigma^{+}}-\frac{1+\left(f_{x}^{-}\right)^{2}}{2 C^{-}\left(f_{\psi}^{-}\right)^{2}} e^{-2 \sigma^{-}}+\lambda\left(\frac{1}{C^{+}}-\frac{1}{C^{-}}\right) f^{+}=\frac{1}{2}\left(\frac{1}{C^{+}}-\frac{1}{C^{-}}\right)
\end{gathered}
$$

and at $\psi=-\psi_{0}^{-}$,

$$
f^{-}=-h^{-}=-C^{-} \ln \left(1+\psi_{0}^{-}\right)
$$

where $\sigma^{ \pm}=\ln \rho^{ \pm}$. It is straightforward to check that

$$
f_{0}(\psi)=-C^{ \pm} \ln (1-\psi), \quad \sigma_{0}(\psi)=\ln \left(\frac{1-\psi}{C^{ \pm}}\right)
$$

are equilibrium solutions of (19) to (24). We shall show that near the equilibrium solutions $f_{0}, \sigma_{0}$, there exists a solitary wave solution of (19) to (24). Therefore, we write

$$
f(x, \psi)=f_{0}(\psi)+w(x, \psi), \quad \sigma(x, \psi)=\sigma_{0}(\psi)+\zeta(x, \psi) .
$$

We use (26) to rewrite (19)-(24) as follows: in $0<\psi<\psi_{0}^{+}$and $-\psi_{0}^{-}<\psi<0$,

$$
\begin{gathered}
w_{\psi \psi}-(1-\psi)^{-1} w_{\psi}+\left(C^{ \pm} /(1-\psi)\right) \zeta_{\psi}+\left(C^{ \pm} /(1-\psi)\right)^{2} w_{x x} \\
=2\left(1+w_{x}\right)^{-2} w_{x} w_{x \psi}\left(w_{\psi}+\left(C^{ \pm} /(1-\psi)\right)\right) \\
\quad-w_{x x}\left[\left(1+w_{x}^{2}\right)^{-1} \times\left(w_{\psi}+\left(C^{ \pm} /(1-\psi)\right)\right)^{2}-\left(C^{ \pm} /(1-\psi)\right)^{2}\right] \\
\quad-w_{\psi} \zeta_{\psi}+w_{x} \zeta_{x}\left(1+w_{x}^{2}\right)^{-1}\left(w_{\psi}+\left(C^{ \pm} /(1-\psi)\right)\right)^{2} \\
\triangleq Z_{0}(w, \zeta), \\
\zeta^{ \pm}=\left(1-C^{ \pm} \lambda\right)^{-1}\left[\lambda w-\left((1-\psi) / C^{ \pm}\right) w_{\psi}+2 \zeta w_{\psi}\left((1-\psi) / C^{ \pm}\right)\right. \\
\quad+(1 / 2)\left(e^{-2 \zeta}-1+2 \zeta+e^{-2 \zeta} w_{x}^{2}\right)\left(1+\left((1-\psi) / C^{ \pm}\right) w_{\psi}\right)^{-2} \\
\quad+(1 / 2)(1-2 \zeta)\left(\left(1+\left((1-\psi) / C^{ \pm}\right) w_{\psi}\right)^{-2}\right. \\
\left.\left.\quad-1+2 w_{\psi}\left((1-\psi) / C^{ \pm}\right)\right)\right] \\
\triangleq\left(1-C^{ \pm} \lambda\right)^{-1}\left(\lambda w-\left((1-\psi) / C^{ \pm}\right) w_{\psi}\right)+Z_{1}(w, \zeta) \triangleq \tilde{Z}_{1} ;
\end{gathered}
$$

at $\psi=\psi_{0}^{+}$,

$$
w^{+}=0
$$


at $\psi=0$,

$$
\begin{gathered}
w^{+}=w^{-} \\
-\frac{\zeta^{+}}{C^{+}}-\frac{w_{\psi}^{+}}{\left(C^{+}\right)^{2}}+\frac{\zeta^{-}}{C^{-}}+\frac{w_{\psi}^{-}}{\left(C^{-}\right)^{2}}+\lambda\left(\frac{1}{C^{+}}-\frac{1}{C^{-}}\right) w \\
=-\left(2 /\left(C^{+}\right)^{2}\right) \zeta^{+} w_{\psi}^{+}+\left(2 /\left(C^{-}\right)^{2}\right) \zeta^{-} w_{\psi}^{-} \\
-\left(1 / 2 C^{+}\right)\left(1+\left(w_{\psi}^{+} / C^{+}\right)\right)^{-2}\left(e^{-2 \zeta^{+}}-1+2 \zeta^{+}+w_{x}^{2} e^{-2 \zeta^{+}}\right) \\
+\left(1 / 2 C^{-}\right)\left(1+\left(w_{\psi}^{-} / C^{-}\right)\right)^{-2}\left(e^{-2 \zeta^{-}}-1+2 \zeta^{-}+w_{x}^{2} e^{-2 \zeta^{-}}\right) \\
-\left(1 / 2 C^{+}\right)\left(\left(1+\left(w_{\psi}^{+} / C^{+}\right)\right)^{-2}-1+2\left(w_{\psi}^{+} / C^{+}\right)\right)\left(1-2 \zeta^{+}\right) \\
+\left(1 / 2 C^{-}\right)\left(1-2 \zeta^{-}\right)\left(\left(1+\left(w_{\psi}^{-} / C^{-}\right)\right)^{-2}-1+2\left(w_{\psi}^{-} / C^{-}\right)\right) \\
\triangleq
\end{gathered}
$$

and at $\psi=-\psi_{0}^{-}$,

$$
w^{-}=0
$$

Here, we remark in passing that $w, \zeta$ mean $w^{+}, \zeta^{+}$in the upper layer and $w^{-}, \zeta^{-}$ in the lower layer. We substitute (28) for $\zeta^{ \pm}$into the left-hand sides of (27), (31), which become

$$
\begin{gathered}
-\frac{C^{ \pm} \lambda}{1-C^{ \pm} \lambda} w_{\psi \psi}+\frac{2 C^{ \pm} \lambda}{\left(1-C^{ \pm} \lambda\right)(1-\psi)} w_{\psi}+\left(\frac{C^{ \pm}}{1-\psi}\right)^{2} w_{x x} \\
=-\left(\frac{C^{ \pm}}{1-\psi}\right) Z_{1 \psi}(w, \zeta)+Z_{0}(w, \zeta) \triangleq F_{0}(w, \zeta) \\
\frac{\lambda}{C^{+}\left(1-C^{+} \lambda\right)} w_{\psi}^{+}-\frac{\lambda}{C^{-}\left(1-C^{-} \lambda\right)} w_{\psi}^{-}-\frac{\lambda^{3}\left(C^{+}-C^{-}\right)}{\left(1-C^{+} \lambda\right)\left(1-C^{-\lambda}\right)} w \\
=\frac{Z_{1}^{+}(w, \zeta)}{C^{+}}-\frac{Z_{1}^{-}(w, \zeta)}{C^{-}}+Z_{2}(w, \zeta) \\
\triangleq G_{0}(w, \zeta) \text { at } \psi=0 .
\end{gathered}
$$

Finally we use (28) to transform (33), (34), (29), (30) and (32) into

$$
\begin{aligned}
& \frac{\left((1-\psi)^{2} w_{\psi}\right)_{\psi}}{C^{ \pm}\left(1-C^{ \pm} \lambda\right)}-\frac{w_{x x}}{\lambda}=-\frac{(1-\psi)^{2}}{\lambda\left(C^{ \pm}\right)^{2}} F_{0}(w, \zeta) \triangleq F_{1}(w, \zeta), \\
& \zeta=\tilde{Z}_{1}(w, \zeta) \triangleq F_{2}(w, \zeta) \quad \text { in } 0<\psi<\psi_{0}^{+},-\psi_{0}^{-}<\psi<0 \\
& w^{+}=0 \quad \text { at } \psi=\psi_{0}^{+}<1 ; \\
& \quad \frac{w_{\psi}^{+}}{C^{+}\left(1-C^{+} \lambda\right)}-\frac{w_{\psi}^{-}}{C^{-}\left(1-C^{-} \lambda\right)}-\frac{\lambda^{2}\left(C^{+}-C^{-}\right)}{\left(1-C^{+} \lambda\right)\left(1-C^{-} \lambda\right)} w=\frac{G_{0}}{\lambda} \triangleq G_{1}(w, \zeta), \\
& w^{+}=w^{-} \quad \text { at } \psi=0 \\
& w^{-}=0 \quad \text { at } \psi=-\psi_{0}^{-} .
\end{aligned}
$$

In the following we shall show that (35) to (40) possess a solitary wave solution under certain conditions. 


\section{Formal derivation}

In this section, we use a formal asymptotic method to derive a solitary wave solution. Let $w, \zeta$ have the following asymptotic expansions

$$
\Phi=\epsilon \Phi_{1}+\epsilon^{2} \Phi_{2}+\cdots,
$$

$\lambda=\lambda_{0}+\epsilon \lambda_{1}$ and $x$ is replaced by $\epsilon^{-1 / 2} x$ under the so-called long-wave assumption. After substituting these expansions into (35) to (40), we obtain a sequence of equations and boundary conditions for the successive approximations by equating the coefficients of like powers of $\epsilon$ on both sides of the equations. The equations for the first-order approximations are

$$
\begin{aligned}
& \left((1-\psi)^{2} w_{1 \psi}\right)_{\psi}=0, \quad \zeta_{1}=\left(1-C^{ \pm} \lambda_{0}\right)^{-1}\left(\lambda_{0} w_{1}-\left((1-\psi) / C^{ \pm}\right) w_{1 \psi}\right) \\
& \quad \text { in } 0<\psi<\psi_{0}^{+},-\psi_{0}^{-}<\psi<0 ; \\
& w_{1}^{+}=0 \text { at } \psi=\psi_{0}^{+}, \quad w_{1}^{-}=0 \text { at } \psi=-\psi_{0}^{-} ; \\
& \frac{w_{1 \psi}^{+}}{C^{+}\left(1-C^{+} \lambda_{0}\right)}-\frac{w_{1 \psi}^{-}}{C^{-}\left(1-C^{-} \lambda_{0}\right)}-\frac{\lambda_{0}^{2}\left(C^{+}-C^{-}\right)}{\left(1-C^{+} \lambda_{0}\right)\left(1-C^{-} \lambda_{0}\right)} w_{1}=0, \\
& w_{1}^{+}=w_{1}^{-} \quad \text { at } \psi=0 .
\end{aligned}
$$

We can easily find a nontrivial solution $w_{1}(x, \psi)=\eta(x) e_{0}(\psi)$ with

$$
e_{0}(\psi)= \begin{cases}\left(\psi_{0}^{+}-\psi\right) \psi_{0}^{-} /(1-\psi) & \text { for } 0<\psi<\psi_{0}^{+}, \\ \left(\psi+\psi_{0}^{-}\right) \psi_{0}^{+} /(1-\psi) & \text { for }-\psi_{0}^{-}<\psi<0\end{cases}
$$

and $\zeta_{1}(x, \psi)=\left(1-C^{ \pm} \lambda_{0}\right)^{-1} \eta(x)\left(\lambda_{0} e_{0}(\psi)-\left((1-\psi) / C^{ \pm}\right) e_{0 \psi}\right)$ if $\lambda_{0}$ satisfies

$$
\begin{aligned}
\lambda^{2}\left(C^{+}-C^{-}\right)+\lambda & \left(-\mu\left(1+\frac{1}{\psi_{0}^{-}}\right)+\frac{1}{\mu}\left(1-\frac{1}{\psi_{0}^{+}}\right)\right) \\
& +\frac{1}{C^{-}}\left(1+\frac{1}{\psi_{0}^{-}}\right)+\frac{1}{C^{+}}\left(\frac{1}{\psi_{0}^{+}}-1\right)=0,
\end{aligned}
$$

which has two roots

$$
\lambda_{+,-}=\frac{\mu\left(1+\left(\psi_{0}^{-}\right)^{-1}\right)+\left(\mu \psi_{0}^{+}\right)^{-1}\left(1-\psi_{0}^{+}\right) \pm \sqrt{\Delta}}{2\left(C^{+}-C^{-}\right)}>0
$$

where $\mu=\left(C^{+} / C^{-}\right)>1, \lambda_{+}>\lambda_{-}$,

$$
\Delta=\left(\mu\left(1+\left(\psi_{0}^{-}\right)^{-1}\right)-\left(\mu \psi_{0}^{+}\right)^{-1}\left(1-\psi_{0}^{+}\right)-2\right)^{2}+4\left(\psi_{0}^{+} \psi_{0}^{-}\right)^{-1}>0
$$

and $\left(\lambda_{ \pm}\right)^{-1 / 2}$ are called critical speeds. It is easy to check that $1-C^{ \pm} \lambda_{ \pm} \neq 0$ for $\mu>1$, $\psi_{0}^{-}>0$ and $\psi_{0}^{+}<1$. Let $\lambda_{0}$ be equal to one of $\lambda_{ \pm}$. In order to find $\eta(x)$, we need the equations for the second-order approximations. In $-\psi_{0}^{-}<\psi<0,0<\psi<\psi_{0}^{+}$,

$$
\begin{gathered}
\frac{\left((1-\psi)^{2} w_{2 \psi}\right)_{\psi}}{C^{ \pm}\left(1-C^{ \pm} \lambda_{0}\right)}=\frac{w_{1 x x}}{\lambda_{0}}-\frac{(1-\psi)^{2}}{\lambda_{0}\left(C^{ \pm}\right)^{2}\left(1-C^{ \pm} \lambda_{0}\right)}\left\{-w_{1 \psi}\left(\lambda_{0} w_{1 \psi}-\left(\frac{(1-\psi) w_{1 \psi}}{C^{ \pm}}\right)_{\psi}\right)\right. \\
-\frac{C^{ \pm}}{1-\psi}\left[\left(\frac{\lambda w_{1} C^{ \pm}-(1-\psi) w_{1 \psi}}{\left(1-C^{ \pm} \lambda_{0}\right) C^{ \pm}}\right)_{\psi}^{2}+\left(\frac{3}{2}\right)\left(\frac{(1-\psi) w_{1 \psi}}{C^{ \pm}}\right)_{\psi}^{2}\right. \\
\left.\left.+2\left(\frac{(1-\psi)\left(\lambda_{0} C^{ \pm} w_{1}-(1-\psi) w_{1 \psi}\right) w_{1 \psi}}{\left(C^{ \pm}\right)^{2}\left(1-C^{ \pm} \lambda_{0}\right)}\right)_{\psi}\right]\right\} \triangleq \tilde{F}
\end{gathered}
$$

at $\psi=\psi_{0}^{+}, w_{2}^{+}=w_{2}^{-}$;

at $\psi=0, w_{2}^{+}=w_{2}^{-}$, 


$$
\begin{aligned}
& \frac{w_{2 \psi}^{+}}{C^{+}\left(1-C^{+} \lambda_{0}\right)}-\frac{w_{2 \psi}^{-}}{C^{-}\left(1-C^{-} \lambda_{0}\right)}-\frac{\lambda_{0}^{2}\left(C^{+}-C^{-}\right)}{\left(1-C^{+} \lambda_{0}\right)\left(1-C^{-} \lambda_{0}\right)} w_{2}=-\frac{w_{1 \psi}^{+} \lambda_{1}}{\left(1-C^{+} \lambda_{0}\right)^{2}} \\
& +\frac{w_{1 \psi}^{-} \lambda_{1}}{\left(1-C^{-} \lambda_{0}\right)^{2}}+\frac{\left(C^{+}-C^{-}\right) \lambda_{1} \lambda_{0}}{\left(1-C^{+} \lambda_{0}\right)^{2}\left(1-C^{-} \lambda_{0}\right)^{2}} w_{1}\left(2-\left(C^{+}+C^{-}\right) \lambda_{0}\right) \\
& -\frac{2 w_{1 \psi}^{+}\left(\lambda_{0} C^{+} w_{1}^{+}-w_{1 \psi}^{+}\right)}{\left(C^{+}\right)^{3}\left(1-C^{+} \lambda_{0}\right) \lambda_{0}}+\frac{2 w_{1 \psi}^{-}\left(\lambda_{0} C^{-} w_{1}^{-}-w_{1 \psi}^{-}\right)}{\left(C^{-}\right)^{3}\left(1-C^{-} \lambda_{0}\right) \lambda_{0}}-\frac{3\left(w_{1 \psi}^{+}\right)^{2}}{2\left(C^{+}\right)^{3}} \\
& +\frac{3\left(w_{1 \psi}^{-}\right)^{2}}{2\left(C^{-}\right)^{3}}-\frac{1}{\left(C^{+}\right)^{3}}\left(\frac{\lambda_{0} C^{+} w_{1}^{+}-w_{1 \psi}^{+}}{1-C^{+} \lambda_{0}}\right)^{2}+\frac{1}{\left(C^{-}\right)^{3}}\left(\frac{\lambda_{0} C^{-} w_{1}^{-}-w_{1 \psi}^{-}}{1-C^{-} \lambda_{0}}\right)^{2} \\
& +\frac{1}{C^{+}\left(1-C^{+} \lambda_{0}\right)}\left[\left(\frac{\lambda_{0} C^{+} w_{1}^{+}-w_{1 \psi}^{+}}{C^{+}\left(1-C^{+} \lambda_{0}\right)}\right)^{2}+\frac{3}{2}\left(\frac{w_{1 \psi}^{+}}{C^{+}}\right)^{2}+\frac{2\left(\lambda_{0} C^{+} w_{1}^{+}-w_{1 \psi}^{+}\right) w_{1 \psi}^{+}}{\left(C^{+}\right)^{2}\left(1-C^{+} \lambda_{0}\right)}\right] \\
& -\frac{1}{C^{-}\left(1-C^{-} \lambda_{0}\right)}\left[\left(\frac{\lambda_{0} C^{-} w_{1}^{-}-w_{1 \psi}^{-}}{C^{-}\left(1-C^{-} \lambda_{0}\right)}\right)^{2}+\frac{3}{2}\left(\frac{w_{1 \psi}^{-}}{C^{-}}\right)^{2}+\frac{2\left(\lambda_{0} C^{-} w_{1}^{-}-w_{1 \psi}^{-}\right) w_{1 \psi}^{-}}{\left(C^{-}\right)^{2}\left(1-C^{-} \lambda_{0}\right)}\right] \\
& \triangleq \tilde{G} ;
\end{aligned}
$$

at $\psi=-\psi_{0}^{-}, w_{2}^{-}=0$.

This is a nonhomogeneous boundary-value problem. Since $e_{0}(\psi)$ is a nontrivial solution for the corresponding homogeneous problem, to solve the nonhomogeneous problem, the nonhomogeneous terms $\tilde{F}$ and $\tilde{G}$ must satisfy the solvability condition

$$
\int_{-\psi_{0}^{-}}^{0} \tilde{F}^{-} e_{0}^{-}(\psi) d \psi+\int_{0}^{\psi_{0}^{+}} \tilde{F}^{+} e_{0}^{+}(\psi) d \psi+\tilde{G} e_{0}(0)=0 .
$$

From (43), we obtain the following equation for $\eta(x)$,

$$
A_{0} \eta_{x x}+\lambda_{1} A_{1} \eta+A_{2} \eta^{2}=0
$$

where

$$
\begin{aligned}
A_{0}= & \frac{1}{\lambda_{0}} \int_{-\psi_{0}^{-}}^{\psi_{0}^{+}} e_{0}^{2}(\psi) d \psi \\
= & \left(\psi_{0}^{+}\right)^{2}\left(\left(\psi_{0}^{-}\right)^{2}+2 \psi_{0}^{-}-2\left(1+\psi_{0}^{-}\right) \ln \left(1+\psi_{0}^{-}\right)\right) \lambda_{0}^{-1} \\
& \quad+\left(\psi_{0}^{-}\right)^{2}\left(2 \psi_{0}^{+}-\left(\psi_{0}^{+}\right)^{2}+2\left(1-\psi_{0}^{+}\right) \ln \left(1-\psi_{0}^{+}\right)\right)^{-1} \lambda_{0}>0, \\
A_{1}= & \frac{\psi_{0}^{-}\left(1-C^{-} \lambda_{0}\right)^{2}+\psi_{0}^{+}\left(1-C^{+} \lambda_{0}\right)^{2}}{\left(1-C^{-} \lambda_{0}\right)^{2}\left(1-C^{+} \lambda_{0}\right)^{2}}>0,
\end{aligned}
$$

and $A_{2}$ is complicated and given in Appendix 1. If $A_{2} \neq 0$, then (44) has a solution

$$
S(x)=\left(-\frac{3 \lambda_{1} A_{1}}{2 A_{2}}\right) \operatorname{sech}^{2}\left(\left(-\lambda_{1} A_{1} / A_{0}\right)^{1 / 2} x / 2\right),
$$

provided that $\lambda_{1}<0$. Thus the solitary wave has to move at a speed greater than a critical speed. The first-order approximation of a solution $(w, \zeta)$ for $(35)$ to $(40)$ is

$$
\begin{aligned}
w(x, \psi) & =\epsilon e_{0}(\psi) S(x)+O\left(\epsilon^{2}\right), \\
\zeta(x, \psi) & =\epsilon\left(1-C^{ \pm} \lambda_{0}\right)^{-1} S(x)\left(\lambda_{0} e_{0}(\psi)-\left((1-\psi) / C^{ \pm}\right) e_{0 \psi}\right)+O\left(\epsilon^{2}\right) .
\end{aligned}
$$

Now we have formally derived a solitary wave solution (46) for (35) to (40). In the following sections, we shall rigorously show that (46) is a first-order approximation of 
a solution of (35) to (40) for small $\epsilon>0$, if $\lambda=\lambda_{0}+\lambda_{1} \epsilon$ with $\lambda_{1}<0$ where $\lambda_{0}=\lambda_{+}$. Note that by choosing some special values of $\mu, \psi_{0}^{+}<1$ and $\psi_{0}^{-}>0$, we obtain

$$
1-C^{ \pm} \lambda_{+}<0, \quad 1-C^{+} \lambda_{-}<0, \text { and } 1-C^{-} \lambda_{-}>0 .
$$

Since $1-C^{ \pm} \lambda_{ \pm} \neq 0$, these inequalities remain for all $\mu>1, \psi_{0}^{+}<1$, and $\psi_{0}^{-}>0$. From (35), we see that the linear part of (35) is elliptic if and only if $1-C^{ \pm} \lambda_{0}<0$. If $\lambda=\lambda_{-}$in (35), the linear part of (35) becomes hyperbolic in the lower layer. Therefore, in what follows we only choose $\lambda_{0}=\lambda_{+}$. Furthermore, $1-C^{-} \lambda_{-}>0$ implies $\lambda_{-}^{-1 / 2}>\left(C^{-}\right)^{1 / 2}$. If the wave speed is near but greater than $\lambda_{-}^{-1 / 2}$, the flow in the lower layer will be supersonic and the governing equation in the physical plane also will become hyperbolic for small $\epsilon$.

\section{Transformations and Banach spaces}

In (35) to (40), we let $\lambda=\lambda_{0}+\lambda_{1} \epsilon=\lambda_{+}+\lambda_{1} \epsilon$ where $\lambda_{+}$is given in (42). Then (35) and (39) become

$$
\begin{gathered}
\frac{\left((1-\psi)^{2} w_{\psi}\right)_{\psi}}{C^{ \pm}\left(1-C^{ \pm} \lambda_{0}\right)}-\frac{w_{x x}}{\lambda_{0}}=-\left(\frac{\left((1-\psi)^{2} w_{\psi}\right)_{\psi} \lambda_{1} \epsilon}{\left(1-C^{ \pm} \lambda_{0}\right)}+\frac{\lambda_{1} \epsilon}{\lambda_{0}} w_{x x}\right)+F_{1}(w, \zeta) \\
\triangleq F_{3}(w, \zeta), \\
\frac{w_{\psi}^{+}}{C^{+}\left(1-C^{+} \lambda_{0}\right)}-\frac{w_{\psi}^{-}}{C^{-}\left(1-C^{-} \lambda_{0}\right)}-\frac{\lambda_{0}^{2}\left(C^{+}-C^{-}\right)}{\left(1-C^{+} \lambda_{0}\right)\left(1-C^{-} \lambda_{0}\right)} w \\
=\frac{w_{\psi}^{-}}{\left(1-C^{-} \lambda_{0}\right)^{2}} \lambda_{1} \epsilon-\frac{w_{\psi}^{+}}{\left(1-C^{+} \lambda_{0}\right)^{2}} \lambda_{1} \epsilon \\
\quad+\frac{\left(C^{+}-C^{-}\right)\left(2 \lambda_{0} \lambda_{1}-\lambda_{0}^{2} \lambda_{1}\left(C^{+}+C^{-}\right)\right)}{\left(1-C^{+} \lambda_{0}\right)^{2}\left(1-C^{-} \lambda_{0}\right)^{2}} w \epsilon+G_{1}(w, \zeta) \\
\triangleq G_{2}(w, \zeta) \quad \text { at } \psi=0
\end{gathered}
$$

Since we need to prove that (46) is an approximation of a solution of (35) to (40), we write

$$
\begin{aligned}
w(x, \psi)= & \epsilon\left(e_{0}(\psi)\left(S\left(\epsilon^{1 / 2} x\right)+\omega(x)\right)+\theta(x, \psi)\right), \\
\zeta(x, \psi)=\epsilon & \left(\left(1-C^{ \pm} \lambda_{0}\right)^{-1}\left(\lambda_{0} e_{0}(\psi)-\left((1-\psi) / C^{ \pm}\right) e_{0 \psi}\right)\right. \\
& \left.\times\left(S\left(\epsilon^{1 / 2} x\right)+w(x)\right)+\xi(x, \psi)\right) .
\end{aligned}
$$

Then the equations (36)-(38), (40), (47), and (48) become

$$
\begin{gathered}
\frac{\left((1-\psi)^{2} \theta_{\psi}\right)_{\psi}}{C^{ \pm}\left(1-C^{ \pm} \lambda_{0}\right)}-\frac{\theta_{x x}}{\lambda_{0}}=\lambda_{0}^{-1}(S+\omega)_{x x} e_{0}+\epsilon^{-1} F_{3}\left(\epsilon\left((S+\omega) e_{0}+\theta\right)\right. \\
\left.\epsilon\left(\left(1-C^{ \pm} \lambda_{0}\right)^{-1}\left(\lambda_{0} e_{0}-\left((1-\psi) / C^{ \pm}\right) e_{0 \psi}\right)(S+\omega)+\xi\right)\right) \\
\triangleq F_{4}(\omega, \theta, \xi) \\
\left.\xi(x, \psi)=-\left(1-C^{ \pm} \lambda_{0}\right)^{-1}\left(\lambda_{0} e_{0}-\left((1-\psi) / C^{ \pm}\right)\right) e_{0 \psi}\right)(S+\omega) \\
+\epsilon^{-1} F_{2}\left(\epsilon\left((S+\omega) e_{0}+\theta\right),\right. \\
\left.\quad \epsilon\left(\left(1-C^{ \pm} \lambda_{0}\right)^{-1}\left(\lambda_{0} e_{0}-\left((1-\psi) / C^{ \pm}\right) e_{0 \psi}\right)(S+\omega)+\xi\right)\right) \\
\triangleq F_{5}(\omega, \theta, \xi) \quad \text { in } \quad 0<\psi<\psi_{0}^{+},-\psi_{0}^{-}<\psi<0
\end{gathered}
$$




$$
\begin{aligned}
& \theta^{+}=0 \quad \text { at } \quad \psi=\psi_{0}^{+}, \quad \theta^{-}=0 \quad \text { at } \psi=-\psi_{0}^{-} ; \\
& \frac{\theta_{\psi}^{+}}{C^{+}\left(1-C^{+} \lambda_{0}\right)}-\frac{\theta_{\psi}^{-}}{C^{-}\left(1-C^{-} \lambda_{0}\right)}-\frac{\lambda_{0}^{2}\left(C^{+}-C^{-}\right) \theta}{\left(1-C^{+} \lambda_{0}\right)\left(1-C^{-} \lambda_{0}\right)} \\
& =\epsilon^{-1} G_{2}\left(\epsilon\left((S+\omega) e_{0}+\theta\right),\right. \\
& \left.\quad \epsilon\left(\left(1-C^{ \pm} \lambda_{0}\right)^{-1}\left(\lambda_{0} e_{0}-\left((1-\psi) / C^{ \pm}\right) e_{0 \psi}\right)(S+\omega)+\xi\right)\right) \\
& \triangleq G_{3}(\omega, \theta, \xi), \quad \text { at } \psi=0 .
\end{aligned}
$$

However, we note that $e_{0}(\psi)$ is a nontrivial solution of the corresponding homogeneous equations for (51), (53)-(55). To solve the nonhomogeneous problem, the nonhomogeneous terms $F_{4}$ and $G_{3}$ must satisfy

$$
\int_{-\psi_{0}^{-}}^{\psi_{0}^{+}} F_{4}(\omega, \theta, \xi) e_{0}(\psi) d \psi+G_{3}(\omega, \theta, \xi) e_{0}(0)=0
$$

From (56) and the expressions of $F_{4}$ and $G_{3}$, and some tedious but straightforward calculations, we obtain an equation for $\omega$

$$
A_{0} \omega_{x x}+\lambda_{1} \epsilon A_{1} \omega+2 \epsilon A_{2} S\left(\epsilon^{1 / 2} x\right) \omega=\epsilon N(\omega, \theta, \xi)
$$

where $N$ includes all the small terms and nonlinear terms and is not presented. In Section 6 , we shall show that $(51)-(55)$ and $(57)$ have a solution $(\omega, \theta, \xi)$ for small $\epsilon>0$.

We conclude this section with the definition of some Banach spaces to be used later. Let $0<\lambda<1$ be fixed, $m, n$ be nonnegative integers,

$$
\begin{aligned}
& D^{+}=\left\{(x, \psi) \mid 0<\psi<\psi_{0}^{+},-\infty<x<+\infty\right\}, \\
& D^{-}=\left\{(x, \psi) \mid-\psi_{0}^{-}<\psi<0,-\infty<x<+\infty\right\}, \\
& \mathcal{H}^{ \pm} f(x, \psi) \triangleq \sup _{\substack{(x, \psi) \in D^{ \pm} \\
|\delta| \leq \delta^{\prime}}}\left(\left|f\left(x+\delta, \psi_{1}\right)-f\left(x, \psi_{2}\right)\right| \exp \left(d \epsilon^{1 / 2}|x|\right)\right. \\
&\left.\times\left(\delta^{2}+\left(\psi_{1}-\psi_{2}\right)^{2}\right)^{-\lambda / 2}\right), \\
& \mathcal{C}^{ \pm} f(x, \psi) \triangleq \sup _{(x, \psi) \in D^{ \pm}}\left(|f(x, \psi)| \exp \left(d \epsilon^{1 / 2}|x|\right)\right) .
\end{aligned}
$$

Here $\delta^{\prime}, d>0$ are fixed constants, we may choose, for example, $\delta^{\prime}=10$, and $d$ will be a small constant to be determined later. Then define the Banach spaces

$$
\begin{aligned}
B_{n}=\left\{f(x, \psi) \in C^{n}\left((-\infty,+\infty) \times\left(\left[-\psi_{0}^{-}, 0\right) \cup\left(0, \psi_{0}^{+}\right]\right)\right) \mid\right. \\
\left.\|f\|_{B_{n}}=\sum_{m=0}^{n} \sum_{k=0}^{m} \mathcal{C}^{ \pm}\left(\frac{\partial^{m} f^{ \pm}}{\partial x^{k} \partial \psi^{m-k}}\right)+\sum_{m=0}^{n} \mathcal{H}^{ \pm}\left(\frac{\partial^{n} f^{ \pm}}{\partial x^{m} \partial \psi^{n-m}}\right)<+\infty\right\} .
\end{aligned}
$$

Let $B_{n}^{0}=\left\{f(x, \psi) \in B_{n} \mid f(x, \psi)=f(-x, \psi)\right\}$. We note that $B_{n}$ and $B_{n}^{0}$ also include functions depending upon $x$ only.

\section{Differential equations}

First, let us consider (57). Let

$$
\mathcal{L}(\omega)=\omega_{x x}+\left(\lambda_{1} A_{2} \epsilon / A_{0}\right) \omega+\left(2 A_{2} \epsilon / A_{0}\right) S\left(\epsilon^{1 / 2} x\right) \omega=\epsilon N / A_{0} .
$$


However, if $f(x)$ and $\omega(x)$ are even, the problem

$$
\begin{aligned}
\mathcal{L}(\omega) & =f & & 0<x<+\infty \\
\omega_{x} & =0 & & \text { at } x=0,
\end{aligned}
$$

has been studied in [14] and the solution can be written as $\omega=\mathcal{L}^{-1} f$. We have

Lemma 1. If $f(x) \in B_{n}^{0}$ for $n \geq 0$ and $d$ is small in $B_{n}^{0}$, then

$$
\left\|\mathcal{L}^{-1} f\right\|_{B_{n+2}^{0}} \leq K \epsilon^{-1}\|f\|_{B_{n}^{0}}
$$

where $K$ is independent of $\epsilon$ and $f(x)$.

The proof of Lemma 1 can be found in [14]. By checking terms in $N(\omega, \theta, \xi)$ carefully and using Lemma 1, it is quite straightforward to obtain the following result.

Theorem 1. If $\omega, \theta \in B_{n}^{0}, \xi \in B_{n-1}^{0}$ with $\|\omega\|_{B_{n}^{0}} \leq K \epsilon^{1 / 2},\|\theta\|_{B_{n}^{0}}+\|\xi\|_{B_{n-1}^{0}} \leq K \epsilon$ (or $K \epsilon^{1 / 2}$ ) for $n \geq 2$, then $N(\omega, \theta, \xi) \in B_{n-2}^{0}$,

$$
\left.\|N(\omega, \theta, \xi)\|_{B_{n-2}^{0}} \leq K \epsilon \quad \text { (or } K \epsilon^{1 / 2}\right),
$$

and

$$
\left.\left\|\mathcal{L}^{-1}\left(\epsilon N / A_{0}\right)\right\|_{B_{n}^{0}} \leq K \epsilon \quad \text { (or } K \epsilon^{1 / 2}\right) .
$$

Now let us consider the partial differential equations (51), (53)-(55). First, we discuss the following equations: in $-\infty<x<+\infty,-\psi_{0}^{-}<\psi<0,0<\psi<\psi_{0}^{+}<1$,

$$
-\lambda_{0} \frac{\left((1-\psi)^{2} u_{\psi}\right)_{\psi}}{C^{ \pm}\left(1-C^{ \pm} \lambda_{0}\right)}+u_{x x}=\varphi_{1}(x, \psi)
$$

at $\psi=0$,

$$
\begin{gathered}
u^{+}(x, 0)=u^{-}(x, 0), \\
-\frac{\lambda_{0} u_{\psi}^{+}}{C^{+}\left(1-C^{+} \lambda_{0}\right)}+\frac{\lambda_{0} u_{\psi}^{-}}{C^{-}\left(1-C^{-} \lambda_{0}\right)}+\frac{\lambda_{0}^{3}\left(C^{+}-C^{-}\right) u}{\left(1-C^{+} \lambda_{0}\right)\left(1-C^{-} \lambda_{0}\right)}=\varphi_{2}(x)
\end{gathered}
$$

at $\psi=-\psi_{0}^{-}$,

$$
u^{-}=0
$$

at $\psi=\psi_{0}^{+}$,

$$
u^{+}=0
$$

where $\varphi_{1}(x, \psi) \in B_{n}^{0}$ and $\varphi_{2}(x) \in B_{n+1}^{0}$ for $n \geq 0$. We shall make use of the family of eigenfunctions $v=v(\psi)$ satisfying

$$
\begin{aligned}
& -\frac{\lambda_{0}\left((1-\psi)^{2} v_{\psi}\right)_{\psi}}{C^{ \pm}\left(1-C^{ \pm} \lambda_{0}\right)}-\tau v=0 \text { in }-\psi_{0}^{-}<\psi<0, \quad 0<\psi<\psi_{0}^{+} \\
& v^{+}=v^{-}, \quad-\frac{\lambda_{0} v_{\psi}^{+}}{C^{+}\left(1-C^{+} \lambda_{0}\right)}+\frac{\lambda_{0} v_{\psi}^{-}}{C^{-}\left(1-C^{-} \lambda_{0}\right)}+\frac{\lambda_{0}^{3}\left(C^{+}-C^{-}\right) v}{\left(1-C^{+} \lambda_{0}\right)\left(1-C^{-} \lambda_{0}\right)}=0 \\
& \quad \text { at } \psi=0 ; \\
& v^{-}=0 \quad \text { at } \psi=-\psi_{0}^{-} ; \quad v^{+}=0 \text { at } \psi=\psi_{0}^{+} .
\end{aligned}
$$


First, let us consider the space $L^{2}\left(-\psi_{0}^{-}, \psi_{0}^{+}\right)$with the usual $L^{2}$-inner product. Define an operator in $L^{2}$ by

$$
\mathcal{A}(u) \triangleq-\frac{\lambda_{0}\left((1-\psi)^{2} u_{\psi}\right)_{\psi}}{C^{ \pm}\left(1-C^{ \pm} \lambda_{0}\right)}
$$

with domain

$$
\begin{aligned}
\mathcal{D}(\mathcal{A})=\left\{u \in L^{2}\left(-\psi_{0}^{-}, \psi_{0}^{+}\right) \mid u^{-} \in H^{2}\left(-\psi_{0}^{-}, 0\right), u^{+} \in H^{2}\left(0, \psi_{0}^{+}\right),\right. \\
u^{+}(0)=u^{-}(0), u^{+}\left(\psi_{0}^{+}\right)=u^{-}\left(-\psi_{0}^{-}\right)=0 \\
\left.-\frac{\lambda_{0} u_{\psi}^{+}(0)}{C^{+}\left(1-C^{+} \lambda_{0}\right)}+\frac{\lambda_{0} u_{\psi}^{-}(0)}{C^{-}\left(1-C^{-} \lambda_{0}\right)}+\frac{\lambda_{0}^{3}\left(C^{+}-C^{-}\right) u(0)}{\left(1-C^{+} \lambda_{0}\right)\left(1-C^{-} \lambda_{0}\right)}=0\right\} .
\end{aligned}
$$

Obviously the solutions of (64) to (66) correspond to the eigenfunctions of $\mathcal{A}$ in $L^{2}$ with eigenvalues $\tau$ and $\mathcal{A}$ is closed and densely defined. Also it is easy to obtain that

$$
\begin{aligned}
(\mathcal{A} u, v)= & \int_{-\psi_{0}^{-}}^{0} \frac{\lambda_{0}(1-\psi)^{2} u_{\psi}^{-} v_{\psi}^{-}}{C^{-}\left(1-C^{-} \lambda_{0}\right)} d \psi+\int_{0}^{\psi_{0}^{+}} \frac{\lambda_{0}(1-\psi)^{2} u_{\psi}^{+} v_{\psi}^{+}}{C^{+}\left(1-C^{+} \lambda_{0}\right)} d \psi \\
& +\lambda_{0}^{3} u(0) v(0) \frac{C^{+}-C^{-}}{\left(1-C^{+} \lambda_{0}\right)\left(1-C^{-} \lambda_{0}\right)}
\end{aligned}
$$

for $u, v \in \mathcal{D}(\mathcal{A})$, which shows that $\mathcal{A}$ is symmetric and all eigenvalues of $\mathcal{A}$ are real. If $u \in \mathcal{D}(\mathcal{A})$, then

$$
\begin{aligned}
\left(u^{+}(0)\right)^{2} & =\left(\int_{0}^{\psi_{0}^{+}} u_{\psi}^{+} d \psi\right)^{2} \leq \int_{0}^{\psi_{0}^{+}}\left((1-\psi) u_{\psi}^{+}\right)^{2} d \psi \int_{0}^{\psi_{0}^{+}}(1-\psi)^{-2} d \psi \\
& =\left(1-\psi_{0}^{+}\right)^{-1} \psi_{0}^{+} \int_{0}^{\psi_{0}^{+}}\left((1-\psi) u_{\psi}^{+}\right)^{2} d \psi \\
\left(u^{-}(0)\right)^{2} & =\left(\int_{-\psi_{0}^{-}}^{0} u_{\psi}^{-} d \psi\right)^{2} \leq \int_{-\psi_{0}^{-}}^{0}\left((1-\psi) u_{\psi}^{-}\right)^{2} d \psi \int_{-\psi_{0}^{-}}^{0}(1-\psi)^{-2} d \psi \\
& =\left(1+\psi_{0}^{-}\right)^{-1} \psi_{0}^{-} \int_{-\psi_{0}^{-}}^{0}\left((1-\psi) u_{\psi}^{-}\right)^{2} d \psi
\end{aligned}
$$

By $1-C^{ \pm} \lambda_{0}<0$ and (42),

$$
\begin{aligned}
&(\mathcal{A} u, u) \leq-\left(\frac{\left(1+\psi_{0}^{-}\right) \lambda_{0}}{\psi_{0}^{-} C^{-}\left(C^{-} \lambda_{0}-1\right)}+\frac{\left(1-\psi_{0}^{+}\right) \lambda_{0}}{\psi_{0}^{+} C^{+}\left(C^{+} \lambda_{0}-1\right)}\right. \\
&\left.-\frac{\lambda_{0}^{3}\left(C^{+}-C^{-}\right)}{\left(1-C^{+} \lambda_{0}\right)\left(1-C^{-} \lambda_{0}\right)}\right) u^{2}(0)=0
\end{aligned}
$$

where the equality holds if and only if $u=K_{0} e_{0}(\psi), e_{0}(\psi)$ is defined in (41), and $K_{0}$ is any constant. Thus all eigenvalues of $\mathcal{A}$ are nonpositive. In order to show that the eigenfunctions of $\mathcal{A}$ form a basis of $L^{2}$, it suffices to show that $(\mathcal{A}-\tau)^{-1}$ exists and is compact in $L^{2}$ for some $\tau>0$. But $(\mathcal{A}-\tau) u=f$ is equivalent to solving

$$
\begin{aligned}
& -\frac{\lambda_{0}\left((1-\psi)^{2} u_{\psi}\right)_{\psi}}{C^{ \pm}\left(1-C^{ \pm} \lambda_{0}\right)}-\tau u=f, \quad \text { in }-\psi_{0}^{-}<\psi<0, \quad 0<\psi<\psi_{0}^{+} \\
& -\frac{\lambda_{0} u_{\psi}^{+}}{C^{+}\left(1-C^{+} \lambda_{0}\right)}+\frac{\lambda_{0} u_{\psi}^{-}}{C^{-}\left(1-C^{-} \lambda_{0}\right)}+\frac{\lambda_{0}^{3}\left(C^{+}-C^{-}\right) u}{\left(1-C^{+} \lambda_{0}\right)\left(1-C^{-} \lambda_{0}\right)}=0 \\
& u^{+}=u^{-} \quad \text { at } \psi=0
\end{aligned}
$$




$$
u^{-}=0 \quad \text { at } \psi=-\psi_{0}^{-}, \quad u^{+}=0 \quad \text { at } \psi=\psi_{0}^{+} .
$$

The solution of the equations is obtained easily as

$$
\begin{aligned}
u^{+}(\psi)=A_{0}^{+} & \left(\left(1-\psi_{0}^{+}\right)^{r_{2}^{+}}(1-\psi)^{r_{1}^{+}}-\left(1-\psi_{0}^{+}\right)^{r_{1}^{+}}(1-\psi)^{r_{2}^{+}}\right) \\
& +(1-\psi)^{r_{1}^{+}} \int_{\psi_{0}^{+}}^{\psi} \frac{C^{+}\left(1-C^{+} \lambda_{0}\right) f^{+}(x)}{\lambda_{0}\left(r_{1}^{+}-r_{2}^{+}\right)(1-x)^{r_{1}^{+}+1}} d x \\
& -(1-\psi)^{r_{2}^{+}} \int_{\psi_{0}^{+}}^{\psi} \frac{C^{+}\left(1-C^{+} \lambda_{0}\right) f^{+}(x)}{\lambda_{0}\left(r_{1}^{+}-r_{2}^{+}\right)(1-x)^{r_{2}^{+}+1}} d x \\
u^{-}(\psi)=A_{0}^{-} & \left(\left(1+\psi_{0}^{-}\right)^{r_{2}^{-}}(1-\psi)^{r_{1}^{-}}-\left(1+\psi_{0}^{-}\right)^{r_{1}^{-}}(1-\psi)^{r_{2}^{-}}\right) \\
& +(1-\psi)^{r_{1}^{-}} \int_{-\psi_{0}^{-}}^{\psi} \frac{C^{-}\left(1-C^{-} \lambda_{0}\right) f^{-}(x)}{\lambda_{0}\left(r_{1}^{-}-r_{2}^{-}\right)(1-x)^{r_{1}^{-}+1}} d x \\
& -(1-\psi)^{r_{2}^{-}} \int_{-\psi_{0}^{-}}^{\psi} \frac{C^{-}\left(1-C^{-} \lambda_{0}\right) f^{-}(x)}{\lambda_{0}\left(r_{1}^{-}-r_{2}^{-}\right)(1-x)^{r_{2}^{-}+1}} d x
\end{aligned}
$$

where

$$
\begin{aligned}
& r_{1}^{ \pm} \triangleq \frac{\lambda_{0}+\left(\lambda_{0}^{2}+4 \tau \lambda_{0} C^{ \pm}\left(C^{ \pm} \lambda_{0}-1\right)\right)^{1 / 2}}{-2 \lambda_{0}} \\
& r_{2}^{ \pm} \triangleq \frac{\lambda_{0}-\left(\lambda_{0}^{2}+4 \tau \lambda_{0} C^{ \pm}\left(C^{ \pm} \lambda_{0}-1\right)\right)^{1 / 2}}{-2 \lambda_{0}}
\end{aligned}
$$

Note that $A_{0}^{+}$and $A_{0}^{-}$are two constants determined by the two boundary conditions at $\psi=0$. Since $\tau>0$ is not an eigenvalue of $\mathcal{A}, A_{0}^{+}$and $A_{0}^{-}$are uniquely defined and bounded if $f(x)$ is bounded in $L^{2}$. For $f(\psi) \in L^{2}$, it is easy to show that $u^{ \pm}(\psi)$ are continuously differentiable with their derivatives bounded by the $L^{2}$-norm of $f(\psi)$. Thus, if $f(\psi)$ is in a bounded set of $L^{2}$, then $u(\psi)$ is in a compact set of $L^{2}$, which implies that $(\mathcal{A}-\tau)^{-1}$ is compact for $\tau>0$. Therefore, there are countably infinitely many eigenvalues, and the set of corresponding eigenfunctions is complete in $L^{2}$. In Appendix 2, the asymptotic behavior of these eigenvalues is given. We summarize the results as follows:

Lemma 2. The system of (64)-(66) has solutions $e_{n}(\psi), \tau=\tau_{n}, n=0,1,2, \ldots$, with $\tau_{0}=0>\tau_{1}>\tau_{2}>\ldots$. The eigenfunctions $e_{n}(\psi), n=1,2,3, \ldots$, form an orthonormal basis of $L^{2}\left(\left(-\psi_{0}^{-}, 0\right) \cup\left(0, \psi_{0}^{+}\right)\right)$together with $e_{0}(\psi)$ defined in (41). Also, $\left|\tau_{n}\right| \cong K_{1} n^{2}$ for large $n$ where $K_{1} \geq c_{0}>0$ with $c_{0}$ a fixed number.

Now we can find the solution of (59)-(63). Assume

$$
\int_{-\psi_{0}^{-}}^{0} \varphi_{1}^{-}(x, \psi) e_{0}^{-}(\psi) d \psi+\int_{0}^{\psi_{0}^{+}} \varphi_{1}^{+}(x, \psi) e_{0}^{+}(\psi) d \psi+e_{0}(0) \varphi_{2}(x)=0 .
$$

By Lemma 2,

$$
\varphi_{1}(x, \psi)=\sum_{i=1}^{\infty} a_{i}(x) e_{i}(\psi)
$$

where the convergence is in $L^{2}$-norm and

$$
a_{i}(x)=\int_{-\psi_{0}^{-}}^{0} \varphi_{1}^{-}(x, \psi) e_{i}^{-}(\psi) d \psi+\int_{0}^{\psi_{0}^{+}} \varphi_{1}^{+}(x, \psi) e_{i}^{+}(\psi) d \psi
$$


Then multiplying (59) by $e_{i}(\psi)$ and integrating by parts twice, we have

$$
C_{i x x}(x)+\tau_{i} C_{i}(x)=a_{i}(x)+\varphi_{2}(x) e_{i}(0)
$$

where $C_{0}^{\prime}=0$, and, for $i=1,2, \ldots$,

$$
C_{i}(x)=\int_{-\psi_{0}^{-}}^{0} u^{-}(x, \psi) e_{i}^{-}(\psi) d \psi+\int_{0}^{\psi_{0}^{+}} u^{+}(x, \psi) e_{i}^{+}(\psi) d \psi
$$

If we let $-\tau_{i}=\mu_{i}^{2}$ for $i=1,2, \ldots$,

$$
\begin{aligned}
C_{i}(x) & =-\left(1 / 2 \mu_{i}\right) \int_{-\infty}^{\infty} \exp \left(-\mu_{i}|x-s|\right)\left(a_{i}(x)+\varphi_{2}(x) e_{i}(0)\right) d x \\
& \triangleq \mathcal{G}_{i}\left(a_{i}(x)\right)+\mathcal{G}_{i}\left(\varphi_{2}(x) e_{i}(0)\right) .
\end{aligned}
$$

Now let

$$
\begin{aligned}
u(x, \psi) & \triangleq \sum_{i=1}^{\infty} C_{i}(x) e_{i}(\psi)=u_{1}(x, \psi)+u_{2}(x, \psi) \\
& \triangleq \sum_{i=1}^{\infty} e_{i}(\psi) \mathcal{G}_{i}\left(a_{i}(x)\right)+\sum_{i=1}^{\infty} e_{i}(\psi) \mathcal{G}_{i}\left(\varphi_{2}(x) e_{i}(0)\right)
\end{aligned}
$$

Then $u(x, \psi) \in L^{2}\left(\left(-\psi_{0}^{-}, 0\right) \cup\left(0, \psi_{0}^{+}\right)\right)$for all $x \in \mathbf{R}$.

We first assume $\varphi_{1}(x, \psi) \in B_{0}^{0}$ and $\varphi_{2}(x) \in B_{1}^{0}$ with compact supports in $\left(-8\left(\psi_{0}^{+}+\psi_{0}^{-}\right)-2,8\left(\psi_{0}^{+}+\psi_{0}^{-}\right)+2\right)$ for the $x$-variable. Note that

$$
\sum_{i=1}^{\infty} \int_{-\infty}^{\infty} C_{i}^{2}(x) d x \leq M<+\infty
$$

where $M$ is a fixed constant. Since $\left|\tau_{i}\right| \sim O\left(i^{2}\right)$,

$$
\begin{aligned}
\left\|D_{x}^{j} u_{1}(x, \psi)\right\|_{L^{2}}^{2} & \leq K\left\|\varphi_{1}\right\|_{B_{0}^{0}}, \\
\left\|D_{x}^{j} u_{2}(x, \psi)\right\|_{L^{2}}^{2} & \leq K\left\|\varphi_{2}\right\|_{B_{1}^{0}},
\end{aligned}
$$

for $j=0,1$. We call $v(x, \psi)$ a generalized solution of $(59)-(63)$ if $v^{-}(x, \psi) \in$ $H^{1}\left(\mathbf{R} \times\left(-\psi_{0}^{-}, 0\right)\right), v^{+}(x, \psi) \in H^{1}\left(\mathbf{R} \times\left(0, \psi_{0}^{+}\right)\right)$, and

$$
\begin{aligned}
&-\int_{-\infty}^{\infty} \int_{0}^{\psi_{0}^{+}}\left(\frac{\lambda_{0}(1-\psi)^{2} u_{\psi}^{+} z_{\psi}^{+}}{C^{+}\left(C^{+} \lambda_{0}-1\right)}+u_{x}^{+} z_{x}^{+}\right) d \psi d x \\
&-\int_{-\infty}^{\infty} \int_{-\psi_{0}^{-}}^{0}\left(\frac{\lambda_{0}(1-\psi)^{2} u_{\psi}^{-} z_{\psi}^{-}}{C^{-}\left(C^{-} \lambda_{0}-1\right)}+u_{x}^{-} z_{x}^{-}\right) d \psi d x \\
&+\int_{-\infty}^{\infty} \frac{\lambda_{0}^{3}\left(C^{+}-C^{-}\right) u^{+}(x, 0) z(x, 0)}{\left(1-C^{+} \lambda_{0}\right)\left(1-C^{-} \lambda_{0}\right)} d x \\
&=\int_{-\infty}^{\infty} \int_{-\psi_{0}^{-}}^{\psi_{0}^{+}} \varphi_{1}(x, \psi) z(x, \psi) d \psi d x+\int_{-\infty}^{\infty} \varphi_{2}(x) z(x, 0) d x
\end{aligned}
$$

for all $z^{+}(x, \psi) \in C^{\infty}\left(\mathbf{R} \times\left(-\psi_{0}^{-}, 0\right]\right), z^{-}(x, \psi) \in C^{\infty}\left(\mathbf{R} \times\left[0, \psi_{0}^{+}\right)\right)$with $z^{+}(x, 0)=$ $z^{-}(x, 0), z^{+} \equiv 0$ in a small neighborhood of $\psi=\psi_{0}^{+}, z^{-} \equiv 0$ in a small neighborhood of $\psi=-\psi_{0}^{-}$, and $z(\cdot, \psi) \in C_{0}^{\infty}(\mathbf{R})$. If $u(x, \psi)$ in (70) has only finitely many nonzero terms, then $u(x, \psi)$ is a generalized solution of (73) by construction. Thus, by (71)(73), we have

$$
\left\|D^{j} u^{+}(x, \psi)\right\|_{L^{2}\left(\mathbf{R} \times\left(0, \psi_{0}^{+}\right)\right)}+\left\|D^{j} u^{-}(x, \psi)\right\|_{L^{2}\left(\mathbf{R} \times\left(-\psi_{0}^{-}, 0\right)\right)}
$$




$$
\leq K\left(\left\|\varphi_{1}\right\|_{B_{0}^{0}}+\left\|\varphi_{2}\right\|_{B_{1}^{0}}\right) \quad \text { for } \quad j=0,1 .
$$

For general $u(x, \psi)$, we can use $u_{n}(x, \psi)$ with finitely many terms to approximate $u(x, \psi)$ to obtain the estimate (74). Therefore, $u(x, \psi)$ defined in (70) is a generalized solution for (73). Now we need to have the Hölder estimates for $u(x, \psi)$. Since $u(x, \psi)$ satisfies (73) and $\varphi_{1}(x, \psi) \in B_{n}^{0}$, the interior estimates of Hölder norms for $u(x, \psi)$ and its derivatives up to second order can be obtained by classical elliptic operator theory [6]. Also, we have Dirichlet boundary values at $\psi=-\psi_{0}^{-}$and $\psi=\psi_{0}^{+}$. Thus, since $\varphi_{1} \in B_{1}^{0}$, the Hölder estimates for $u(x, \psi)$ can be extended to $\psi=-\psi_{0}^{-}, \psi=\psi_{0}^{+}$ [6]. Therefore, we only need Hölder estimates at $\psi=0^{ \pm}$where $0^{ \pm}$is denoted as the limit when $\psi \rightarrow 0$ from the positive or negative direction, respectively.

First, we change (73) into a more manageable form for $x$ and $\psi$. Let

$$
\begin{array}{ll}
d^{ \pm}=\left(\frac{C^{ \pm}\left(C^{ \pm} \lambda_{0}-1\right)}{\lambda_{0}}\right)^{1 / 2}, & y=-d^{ \pm} \ln (1-\psi), \\
u^{ \pm}=\sqrt{d^{ \pm}}(1-\psi)^{-1 / 2} v^{ \pm}, & z^{ \pm}=\sqrt{d^{ \pm}}(1-\psi)^{-1 / 2} q^{ \pm} .
\end{array}
$$

Then (73) becomes

$$
\begin{gathered}
\int_{-\infty}^{\infty}\left(\frac{v^{+}(x, 0) q^{+}(x, 0)}{4\left(d^{+}\right)^{2}}-\frac{v^{-}(x, 0) q^{-}(x, 0)}{4\left(d^{-}\right)^{2}}+\frac{\lambda_{0}^{3}\left(C^{+}-C^{-}\right) d^{+} v^{+}(x, 0) q^{+}(x, 0)}{\left(C^{+} \lambda_{0}-1\right)\left(C^{-} \lambda_{0}-1\right)}\right) d x \\
-\int_{-\infty}^{\infty}\left(\int_{0}^{-d^{+} \ln \left(1-\psi_{0}^{+}\right)}+\int_{-d^{-} \ln \left(1+\psi_{0}^{-}\right)}^{0}\right)\left(v_{y}^{ \pm} q_{y}^{ \pm}+v_{x}^{ \pm} q_{x}^{ \pm}\right) d y d x \\
=\int_{-\infty}^{\infty} \varphi_{2}(x)\left(d^{+}\right)^{1 / 2} q^{+}(x, 0) d x+\int_{-\infty}^{\infty}\left(\int_{-d^{-} \ln \left(1+\psi_{0}^{-}\right)}^{0}+\int_{0}^{-d^{+} \ln \left(1-\psi_{0}^{+}\right)}\right) \\
\times\left(v^{ \pm} q^{ \pm} 4\left(d^{ \pm}\right)^{2}+\varphi_{1}^{ \pm}(x, y)\left(d^{ \pm}\right)^{-1 / 2} \exp \left(-\frac{y}{2 d^{ \pm}}\right)\right) q^{ \pm}(x, y) d y d x
\end{gathered}
$$

where $q^{ \pm}(x, y)$ has the same properties as $z^{ \pm}(x, \psi)$ except that at $\psi=0$, $\left(d^{+}\right)^{1 / 2} q^{+}(x, 0)=\left(d^{-}\right)^{1 / 2} q^{-}(x, 0)$. Now let $\psi_{m}=\min \left(-d^{+} \ln \left(1-\psi_{0}^{+}\right), d^{+} \ln \left(1+\psi_{0}^{-}\right)\right)$. We choose different $q^{ \pm}(x, y)$ so that when $y$ is near zero, we can have local Hölder estimates with considerations of the boundary conditions at $y=0$. Note that we need $u^{+}(x, 0)=u^{-}(x, 0)$ or $\sqrt{d^{+}} v^{+}(x, 0)=\sqrt{d^{-}} v^{-}(x, 0)$. Thus, we let $q(x, y)$ be in $C_{0}^{\infty}\left(\mathbf{R} \times\left(-\psi_{m}, 0\right)\right)$ with

$$
q^{-}(x, y)=\sqrt{d^{-}} q(x, y), \quad q^{+}(x, y)=-\sqrt{d^{+}} q(x,-y) .
$$

Thus, the equation (76) becomes

$$
\begin{aligned}
- & \int_{-\infty}^{\infty} \int_{-\psi_{m}}^{0}\left(\left(\sqrt{d^{-}} v^{-}-\sqrt{d^{+} v^{+}}\right)_{y} q_{y}+\left(\sqrt{d^{-}} v^{-}-\sqrt{d^{+}} v^{+}\right)_{x} q_{x}\right) d y d x \\
& =\int_{-\infty}^{\infty} \int_{-\psi_{m}}^{0}\left(\frac{v^{-}}{4\left(d^{-}\right)^{3 / 2}}-\frac{v^{+}}{4\left(d^{+}\right)^{3 / 2}}+\varphi_{1}^{-} e^{-y / 2 d^{-}}-\varphi_{1}^{+} e^{-y / 2 d^{+}}\right) q(x, y) d y d x
\end{aligned}
$$

If we view $\sqrt{d^{-}} v^{-}(x, y)-\sqrt{d^{+}} v^{+}(x,-y)$ as a solution of $(77)$ in $\mathbf{R} \times\left(-\psi_{m}, 0\right)$ with Dirichlet boundary condition at $y=0$, then the Hölder estimates of $\sqrt{d^{-}} v^{-}(x, y)-$ $\sqrt{d^{+}} v^{+}(x,-y)$ and its derivatives up to second order near $y=0$ can be bounded by the Hölder norms of $v^{ \pm}$and $\varphi_{1}^{ \pm}$. Also $\sqrt{d^{-}} v^{-}=\sqrt{d^{+}} v^{+}$can be obtained at $y=0$. Next we choose test functions $q^{ \pm}$where $q^{+}(x, y)=\left(d^{+}\right)^{-1 / 2} q(x,-y)$ and 
$q^{-}(x, y)=\left(d^{-}\right)^{-1 / 2} q(x, y)$ with $q(x, y) \in C^{\infty}\left(\mathbf{R} \times\left(-\psi_{m}, 0\right]\right), q(\cdot, y) \in C_{0}^{\infty}(\mathbf{R})$, and $q(x, \cdot) \equiv 0$ for $y$ near $-\psi_{m}$. Then $(76)$ becomes

$$
\begin{aligned}
& -\int_{-\infty}^{\infty} \int_{-\psi_{m}}^{0}\left[\left(\left(d^{-}\right)^{-1 / 2} v^{-}+\left(d^{+}\right)^{-1 / 2} v^{+}\right)_{y} q_{y}\right. \\
& \left.+\left(\left(d^{-}\right)^{-1 / 2} v^{-}+\left(d^{+}\right)^{-1 / 2} v^{+}\right)_{x} q_{x}\right] d y d x \\
& +\int_{-\infty}^{\infty}\left(\frac{1}{2\left(d^{+}\right)^{2}}-\frac{1}{2\left(d^{-}\right)^{2}}+\frac{\lambda_{0}^{3}\left(d^{+}-d^{-}\right)}{\left(C^{+} \lambda_{0}-1\right)\left(C^{-} \lambda_{0}-1\right)}\right)\left(\frac{1}{d^{+}}+\frac{1}{d^{-}}\right) \\
& \quad \times\left(\left(d^{-}\right)^{-1 / 2} v^{-}(x, 0)+\left(d^{+}\right)^{-1 / 2} v^{+}(x, 0)\right) q(x, 0) d x \\
& =\int_{-\infty}^{\infty} \varphi_{2}(x) q(x, 0) d x+\int_{-\infty}^{\infty} \int_{-\psi_{m}}^{0}\left(\varphi_{1}^{-}\left(d^{-}\right)^{-1} e^{-y /\left(2 d^{-}\right)}-\varphi_{1}^{+}\left(d^{+}\right)^{-1} e^{-y /\left(2 d^{+}\right)}\right. \\
& \left.\quad+\frac{v^{-}}{4\left(d^{-}\right)^{5 / 2}}-\frac{v^{+}}{4\left(d^{+}\right)^{5 / 2}}\right) q(x, y) d y d x
\end{aligned}
$$

This is an elliptic equation for $\left(d^{-}\right)^{-1 / 2} v^{-}+\left(d^{+}\right)^{-1 / 2} v^{+}$in the variational form with an oblique boundary condition

$$
\begin{gathered}
-\left(\frac{v^{+}}{\left(d^{+}\right)^{1 / 2}}+\frac{v^{-}}{\left(d^{-}\right)^{1 / 2}}\right)_{y}+\left(\frac{1}{2\left(d^{+}\right)^{2}}-\frac{1}{2\left(d^{-}\right)^{2}}+\frac{\lambda_{0}^{3}\left(C^{+}-C^{-}\right)}{\left(C^{+} \lambda_{0}-1\right)\left(C^{-} \lambda_{0}-1\right)}\right) \\
\times\left(\frac{1}{d^{+}}+\frac{1}{d^{-}}\right)\left(\left(d^{+}\right)^{-1 / 2} v^{+}+\left(d^{-}\right)^{-1 / 2} v^{-}\right)=\varphi_{2}(x) \quad \text { at } \quad \psi=0 .
\end{gathered}
$$

Thus, we have the Hölder estimates of $\left(d^{+}\right)^{-1 / 2} v^{+}+\left(d^{-}\right)^{-1 / 2} v^{-}$and its derivatives up to second order near $y=0$, and these estimates are bounded by $\left\|\varphi_{2}\right\|_{B_{1}^{0}}$ and $\|v\|_{B_{0}^{0}}+\left\|\varphi_{1}\right\|_{B_{0}^{0}}$. If we transform $v$ and $y$ back to $u$ and $\psi$, we obtain the Hölder estimates of $u^{ \pm}(x, \psi)$ near $\psi=0$. Therefore, if $\varphi_{1} \in B_{0}^{0}$ and $\varphi_{2} \in B_{1}^{0}$, and $\varphi_{1}, \varphi_{2}$ satisfy (67), then $u(x, \psi)$ defined in (70) is in $B_{2}^{0}$, and

$$
\|u(x, \psi)\|_{B_{2}^{0}} \leq K\left(\left\|\varphi_{1}\right\|_{B_{0}^{0}}+\left\|\varphi_{2}\right\|_{B_{1}^{0}}+\|u\|_{B_{0}^{0}}\right) .
$$

Here we have used the compact supports of $\varphi_{1}, \varphi_{2}$ and the exponential decay of the Green's function in (70) as $|x| \rightarrow \infty$. However, by checking the terms in (70),

$$
\|u\|_{B_{0}^{0}} \leq K\left(\left\|\varphi_{1}\right\|_{B_{0}^{0}}+\left\|\varphi_{2}\right\|_{B_{1}^{0}}\right)
$$

since $e_{i}(0)$ is bounded (Appendix 2), and $\left|\tau_{i}\right| \sim O\left(i^{2}\right)$. Finally, $u(x, \psi)$ defined in (70) satisfies

$$
\|u(x, \psi)\|_{B_{2}^{0}} \leq K\left(\left\|\varphi_{1}\right\|_{B_{0}^{0}}+\left\|\varphi_{2}\right\|_{B_{1}^{0}}\right)
$$

if $\varphi_{1}, \varphi_{2}$ satisfy $(67)$.

Next, let us prove (79) in the case that $\varphi_{1}(x, \psi)$ and $\varphi_{2}(x)$ may not have compact supports in the $x$-variable. Let $x_{0}$ be fixed first and choose $x$ such that $\left|x-x_{0}\right|<1$. Define a cut-off function $p(y) \in C^{\infty}(\mathbf{R})$ with $p(y) \equiv 1$ for $|y| \geq 8\left(\psi_{0}^{+}+\psi_{0}^{-}\right)+2$ and $p(y) \equiv 0$ for $|y| \leq 4\left(\psi_{0}^{+}+\psi_{0}^{-}\right)+2$. Write $(70)$ as

$$
\begin{aligned}
u(x, \psi)= & \sum_{i=1}^{\infty} e_{n}(\psi) \mathcal{G}_{i}\left(p\left(s-x_{0}\right)\left(a_{i}(s)+\varphi_{2}(s) e_{i}(0)\right)\right) \\
& \quad+\sum_{i=1}^{\infty} e_{n}(\psi) \mathcal{G}_{i}\left(\left(1-p\left(s-x_{0}\right)\right)\left(a_{i}(s)+\varphi_{2}(s) e_{i}(0)\right)\right) \\
\triangleq & +I I .
\end{aligned}
$$


By the definition of $p(s)$, the integration variable $s$ in $I$ satisfies $|s-x| \geq 4\left(\psi_{0}^{+}+\psi_{0}^{-}\right)+1$. Thus the differentiated series of $I$ is uniformly convergent and derivatives can be taken term by term. By a routine calculation [14],

$$
\|I\|_{B_{2}^{*}} \leq K\left(\|\varphi\|_{B_{0}^{0}}+\left\|\varphi_{2}\right\|_{B_{1}^{0}}\right)
$$

where $K$ is a generic constant independent of $x_{0}$ and $B_{2}^{*}$ is defined just as $B_{2}$ except that the sup-norm is taken only for $\left|x-x_{0}\right|<1$. For $I I$, we write

$$
\begin{aligned}
& \int_{-\infty}^{\infty} \exp \left(-\mu_{i}|x-s|\right)\left(1-p\left(s-x_{0}\right)\right)\left(a_{i}(s)+\varphi_{2}(s) e_{i}(0)\right) d s \\
& \quad=\int_{-\infty}^{\infty} \exp \left(-\mu_{i}\left|x-x_{0}-y\right|\right)(1-p(y))\left(a_{i}\left(y+x_{0}\right)+\varphi_{2}\left(y+x_{0}\right) e_{i}(0)\right) d y .
\end{aligned}
$$

Let $x^{*}=x-x_{0}, a_{i}^{*}(y)=a_{i}\left(y+x_{0}\right)(1-p(y)), \varphi_{2}^{*}(y)=(1-p(y)) \varphi_{2}\left(y+x_{0}\right)$, and $\varphi_{1}^{*}(y, \psi)=(1-p(y)) \varphi_{1}\left(y+x_{0}, \psi\right)$. Then

$$
I I=\sum_{i=1}^{\infty} \mathcal{G}_{i}\left(a_{i}^{*}\right) e_{i}(\psi)+\sum_{i=1}^{\infty} e_{i}(\psi) \mathcal{G}_{i}\left(\varphi_{2}^{*}\right) e_{i}(0) .
$$

$I I$ has the same form as $(70)$, and $\varphi_{1}^{*}, \varphi_{2}^{*}$ have compact supports in $\left(-8\left(\psi_{0}^{+}+\psi_{0}^{-}\right)-2\right.$, $\left.8\left(\psi_{0}^{+}+\psi_{0}^{-}\right)+2\right)$. Therefore, (79) holds, and, in particular,

$$
\|I I\|_{B_{2}^{*}} \leq K\left(\left\|\varphi_{1}^{*}(y, \psi)\right\|_{B_{0}}+\left\|\varphi_{2}^{*}(y)\right\|_{B_{1}}\right)
$$

where $K$ is independent of $x_{0}$. However, note that

$$
\sup _{y \in \mathbf{R}}\left(\left|(1-p(y)) \varphi_{1}\left(y+x_{0}\right)\right|\right) \triangleq \sup _{y \in \mathbf{R}} I I I(y)
$$

where $I I I(y)=\left|(1-p(y)) \varphi_{1}\left(y+x_{0}\right)\right| \exp \left(\left|y+x_{0}\right|\right) \exp \left(-\left|y+x_{0}\right|\right)$, and

$$
\begin{aligned}
\exp \left(-\left|x_{0}\right|+8\left(\psi_{0}^{+}+\psi_{0}^{-}\right)+2\right) & \geq \exp \left(-\left|y+x_{0}\right|\right) \\
& \geq \exp \left(-\left|x_{0}\right|-8\left(\psi_{0}^{+}+\psi_{0}^{-}\right)-2\right),
\end{aligned}
$$

since $I I I(y) \equiv 0$ for $|y| \geq 8\left(\psi_{0}^{+}+\psi_{0}^{-}\right)+2$. Thus,

$$
\|I I\|_{B_{2}^{*}} \exp \left(-d\left|x_{0}\right| \epsilon^{1 / 2}\right) \leq K\left(\left\|\varphi_{1}\right\|_{B_{0}^{0}}+\left\|\varphi_{2}\right\|_{B_{1}^{0}}\right)
$$

where $K$ is independent of $x_{0}$. Since $x_{0}$ is arbitrary and $u(x, \psi)$ is even, we have

$$
\|u(x, \psi)\|_{B_{2}^{0}} \leq K\left(\left\|\varphi_{1}\right\|_{B_{0}^{0}}+\left\|\varphi_{2}\right\|_{B_{1}^{0}}\right) .
$$

By induction, we obtain

$$
\|u(x, \psi)\|_{B_{n+2}^{0}} \leq K\left(\left\|\varphi_{1}\right\|_{B_{n}^{0}}+\left\|\varphi_{2}\right\|_{B_{n+1}^{0}}\right)
$$

for every positive integer $n$. Hence, we have

Theorem 2. If $\varphi_{1} \in B_{n}^{0}, \varphi_{2} \in B_{n+1}^{0}$ and $\varphi_{1}, \varphi_{2}$ satisfy (67), then the solution $u(x, \psi)$ of $(59)-(63)$ exists in $B_{n+2}^{0}$ and satisfies $(80)$.

Now let the solution of (59)-(63) be

$$
u(x, \psi)=\mathcal{P}\left(\varphi_{1}(x, \psi), \varphi_{2}(x)\right) .
$$

Then, we consider (51)-(55) and write

$$
\begin{aligned}
& F_{4}(\omega, \theta, \xi)=M_{1}(\omega)+\tilde{F}_{4}(\omega, \theta, \xi) \\
& G_{3}(\omega, \theta, \xi)=M_{2}(\omega)+\tilde{G}_{3}(\omega, \theta, \xi)
\end{aligned}
$$


where $M_{1}(\omega)$ and $M_{2}(\omega)$ contain only the terms with $\omega_{x x}, \epsilon \omega$, and $\epsilon S\left(\epsilon^{1 / 2} x\right) \omega$ in $F_{4}$ and $G_{3}$, respectively, and $\tilde{F}_{4}$ and $\tilde{G}_{3}$ are the remainders. By the derivation of (51) from (56), we can see that the left-hand side of (57), denoted by $A_{0} \mathcal{L}(\omega)$ in (58), is from $\left(M_{1}, M_{2}\right)$ only, and the right-hand side of $(57)$ is from $\left(\tilde{F}_{4}, \tilde{G}_{3}\right)$. The left-hand side of $(56)$ is equal to $A_{0} \mathcal{L}(\omega)-\epsilon N$. Since $\mathcal{L}^{-1}$ exists by Lemma 1 and Theorem 1 , define

$$
\begin{aligned}
& F(\omega, \theta, \xi) \triangleq M_{1}\left(\mathcal{L}^{-1}\left(\epsilon N / A_{0}\right)\right)+\tilde{F}_{4}(\omega, \theta, \xi), \\
& G(\omega, \theta, \xi) \triangleq M_{2}\left(\mathcal{L}^{-1}\left(\epsilon N / A_{0}\right)\right)+\tilde{G}_{3}(\omega, \theta, \xi),
\end{aligned}
$$

which are equivalent to $F_{4}$ and $G_{3}$ for the problem. If we replace $F_{4}$ and $G_{3}$ in (51) and (55) by $F$ and $G$, respectively, then by a similar derivation of (57) from (56) and noting that $\mathcal{L}^{-1}$ only appears in $\left(M_{1}, M_{2}\right)$, we have

$$
\int_{-\psi_{0}^{-}}^{\psi_{0}^{+}} F(\omega, \theta, \xi) e_{0}(\psi) d \psi+e_{0}(0) G(\omega, \theta, \xi)=A_{0} \mathcal{L}\left(\mathcal{L}^{-1}\left(\epsilon N / A_{0}\right)\right)-\epsilon N=0 .
$$

For such $F$ and $G$, the condition (67) is satisfied automatically. If we assume that $\theta(x, \psi), \omega(x) \in B_{n+2}^{0}$, and $\xi(x, \psi) \in B_{n+1}^{0}$ with $\|\theta\|_{B_{n+2}^{0}}+\|\omega\|_{B_{n+2}^{0}}+\|\xi\|_{B_{n+1}^{0}} \leq K \epsilon^{1 / 2}$ for some $K>0$, then by checking the terms in $F^{ \pm}(\omega, \theta, \xi)$ and using Theorem 1 , we have $F, G \in B_{n}^{0}$ and

$$
\|F\|_{B_{n}^{0}}+\|G\|_{B_{n}^{0}} \leq K \epsilon\left(\|\theta\|_{B_{n+2}^{0}}+\|\omega\|_{B_{n+2}^{0}}+\|\xi\|_{B_{n+1}^{0}}+1\right) .
$$

Thus, by Theorem 2, we obtain

Theorem 3. If $\theta(x, \psi), \omega(x) \in B_{n+2}^{0}$, and $\xi \in B_{n+1}^{0}$ with $\|\theta\|_{B_{n+2}^{0}}+\|\omega\|_{B_{n+2}^{0}}+$ $\|\xi\|_{B_{n+1}^{0}} \leq K \epsilon^{1 / 2}$, the solution of (51) and (53)-(55) with $F_{4}$ and $G_{3}$ replaced by $F$ and $G$, respectively, exists and satisfies

$$
\|\mathcal{P}(F, G)\|_{B_{n+2}^{0}} \leq K \epsilon\left(\|\theta\|_{B_{n+2}^{0}}+\|\omega\|_{B_{n+2}^{0}}+\|\xi\|_{B_{n+1}^{0}}+1\right) .
$$

Finally, by checking the terms of $F_{5}(\omega, \theta, \xi)$ in (52), it can be shown that if $\omega, \theta \in$ $B_{n+2}^{0}$ and $\xi \in B_{n+1}^{0}$ are bounded with respect to their norms, then $F_{5} \in B_{n+1}^{0}$ and

$$
\left\|F_{5}(\omega, \theta, \xi)\right\|_{B_{n+1}^{0}} \leq K\left(\|\theta\|_{B_{n+2}^{0}}+\epsilon\right) .
$$

Now we are ready to obtain the existence of solutions of (51)-(55) and (57).

\section{The existence proof}

First, we invert (57) into

$$
\omega=\left(A_{0}\right)^{-1} \mathcal{L}^{-1}(\epsilon N(\omega, \theta, \xi))
$$

by using Theorem 1 . Then, we consider (51), (53)-(55) with $F_{4}$ and $G_{3}$ replaced by $F$ and $G$ in (84) and (85), respectively. By (86), $F$ and $G$ satisfy the condition (67). Hence, (51), (53)-(55) can be transformed into

$$
\theta(x, \psi)=\mathcal{P}(F(\omega, \theta, \xi), G(\omega, \theta, \xi)) \triangleq \mathcal{T}_{2}(\omega, \theta, \xi),
$$

by using Theorem 2 and (81). Finally, we have

$$
\xi(x, \psi)=F_{5}(\omega, \theta, \xi)
$$

from (52). By Theorem 3, we obtain that if $\|\theta\|_{B_{n+2}^{0}}+\|\omega\|_{B_{n+2}^{0}}+\|\xi\|_{B_{n+1}^{0}} \leq K \epsilon^{1 / 2}$ for $n \geq 0$, then

$$
\left\|\mathcal{T}_{2}(\omega, \theta, \xi)\right\| \leq K \epsilon
$$


In order to use (87) and Theorem 1, we transform (88) and (90) into

$$
\begin{aligned}
\xi(x, \psi) & =F_{5}\left(\omega, \mathcal{T}_{2}(\omega, \theta, \xi), \xi\right) \triangleq \mathcal{T}_{3}(\omega, \theta, \xi) \\
\omega(x) & =A_{0}^{-1} \mathcal{L}^{-1}\left(\epsilon N\left(\omega, \mathcal{T}_{2}(\omega, \theta, \xi), \mathcal{T}_{3}(\omega, \theta, \xi)\right)\right) \\
& \triangleq \mathcal{T}_{1}(\omega, \theta, \xi)
\end{aligned}
$$

Then, by (87), (91), and Theorem 1 , we have

$$
\left\|\mathcal{T}_{3}(\omega, \theta, \xi)\right\|_{B_{n+1}^{0}}+\left\|\mathcal{T}_{1}(\omega, \theta, \xi)\right\|_{B_{n+2}^{0}} \leq K \epsilon
$$

Define a closed convex set in the Banach space $B_{n+2}^{0} \times B_{n+2}^{0} \times B_{n+1}^{0}$ for $n \geq 0$,

$$
\begin{aligned}
\mathcal{S}_{b}=\{X= & (\omega, \theta, \xi) \in B_{n+2}^{0} \times B_{n+2}^{0} \times B_{n+1}^{0} \mid \\
& \left.\|X\|=\|\omega\|_{B_{n+2}^{0}}+\|\theta\|_{B_{n+2}^{0}}+\|\xi\|_{B_{n+1}^{0}} \leq b \epsilon^{1 / 2}\right\} .
\end{aligned}
$$

Let

$$
\mathcal{T}(\omega, \theta, \xi)=\left(\mathcal{T}_{1}(\omega, \theta, \xi), \mathcal{T}_{2}(\omega, \theta, \xi), \mathcal{T}_{3}(\omega, \theta, \xi)\right) .
$$

By (91) and (94), $\mathcal{T}$ maps $\mathcal{S}_{b}$ into itself if $K \epsilon^{1 / 2}<b$ for $\epsilon$ small. Also, by using similar proofs as for Theorems 1 and 3 , we have

Theorem 4. If $X^{(1)}=\left(\omega^{(1)}, \theta^{(1)}, \xi^{(1)}\right)$ and $X^{(2)}=\left(\omega^{(2)}, \theta^{(2)}, \xi^{(2)}\right) \in \mathcal{S}_{b}$, then

$$
\left\|\mathcal{T}\left(X^{(1)}\right)-\mathcal{T}\left(X^{(2)}\right)\right\| \leq K \epsilon^{1 / 2}\left\|X^{(1)}-X^{(2)}\right\| .
$$

We choose $K \epsilon^{1 / 2} \leq 1 / 2$ for smaller $\epsilon>0$ such that $\mathcal{T}$ maps $\mathcal{S}_{b}$ into $\mathcal{S}_{b}$ and is a contraction in $\mathcal{S}_{b}$. The contraction mapping theorem implies the existence of a unique solution in $\mathcal{S}_{b}$ for (89), (92), and (93), which gives the solution of (51)-(55) and (57). Finally, we have the existence theorem.

Theorem 5. If $\lambda=\lambda_{0}+\lambda_{1} \epsilon$ with $\lambda_{1}<0$ and

$$
\lambda_{0}=\frac{\mu\left(1+\left(\psi_{0}^{-}\right)^{-1}\right)+\left(\mu \psi_{0}^{+}\right)^{-1}\left(1-\psi_{0}^{+}\right)+\sqrt{\Delta}}{2\left(C^{+}-C^{-}\right)}
$$

where $\mu=\left(C^{+} / C^{-}\right)>1$ and

$$
\Delta=\left(\mu\left(1+\left(\psi_{0}^{-}\right)^{-1}\right)-\left(\mu \psi_{0}^{+}\right)^{-1}\left(1-\psi_{0}^{+}\right)-2\right)^{2}+4\left(\psi_{0}^{+} \psi_{0}^{-}\right)^{-1}>0,
$$

then for small $\epsilon>0$, there exists a solution of (19)-(24) in the form

$$
\begin{gathered}
f^{+}(x, \psi)=-C^{+} \ln (1-\psi)+\epsilon \psi_{0}^{-}\left(\psi_{0}^{+}-\psi\right)(1-\psi)^{-1}\left(S\left(\epsilon^{1 / 2} x\right)+\omega(x)\right)+\epsilon \theta^{+}(x, \psi), \\
\sigma^{+}(x, \psi)=\ln \rho^{+}(x, \psi)=\ln \frac{(1-\psi)}{C^{+}}+\epsilon\left(\left(1-C^{+} \lambda_{0}\right) C^{+}(1-\psi)\right)^{-1} \\
\quad \times\left(\lambda_{0} C^{+} \psi_{0}^{-}\left(\psi_{0}^{+}-\psi\right)-\psi_{0}^{-}\left(\psi_{0}^{+}-1\right)\right)\left(S\left(\epsilon^{1 / 2} x\right)+\omega(x)\right) \\
\quad+\epsilon \xi^{+}(x, \psi) \quad \text { for } \quad 0<\psi<\psi_{0}^{+}<1, \\
f^{-}(x, \psi)=-C^{-} \ln (1-\psi)+\epsilon \psi_{0}^{+}\left(\psi+\psi_{0}^{-}\right)(1-\psi)^{-1}\left(S\left(\epsilon^{1 / 2} x\right)+\omega(x)\right)+\epsilon \theta^{-}(x, \psi), \\
\sigma^{-}(x, \psi)=\ln \rho^{-}(x, \psi)=\ln \frac{(1-\psi)}{C^{-}}+\epsilon\left(\left(1-C^{-} \lambda_{0}\right) C^{-}(1-\psi)\right)^{-1} \\
\times\left(\lambda_{0} C^{-} \psi_{0}^{+}\left(\psi+\psi_{0}^{-}\right)-\psi_{0}^{+}\left(1+\psi_{0}^{-}\right)\right)\left(S\left(\epsilon^{1 / 2} x\right)+\omega(x)\right) \\
+\epsilon \xi^{-}(x, \psi) \quad \text { for } \quad-\psi_{0}^{-}<\psi<0,
\end{gathered}
$$


where

$$
S\left(\epsilon^{1 / 2} x\right)=\left(\frac{3 \lambda_{1} A_{1}}{2 A_{2}}\right) \operatorname{sech}^{2}\left(\left(-\epsilon \lambda_{1} A_{1} / A_{0}\right)^{1 / 2} x / 2\right),
$$

$A_{0}, A_{1}>0$, and $A_{2} \neq 0$ are defined in (44) and Appendix $1, \omega(x), \theta(x, \theta) \in B_{n+2}^{0}$ and $\xi(x, \psi) \in B_{n+1}^{0}$ for $n \geq 0$ with

$$
\|\omega\|_{B_{n+2}^{0}}+\|\theta\|_{B_{n+2}^{0}}+\|\xi\|_{B_{n+1}^{0}} \leq K \epsilon
$$

and $K$ is independent of $\epsilon$. The interface is given by

$$
\eta(x)=\epsilon \psi_{0}^{-} \psi_{0}^{+} S\left(\epsilon^{1 / 2} x\right)+O\left(\epsilon^{2}\right),
$$

and $\psi_{0}^{+} \psi_{0}^{-} S\left(\epsilon^{1 / 2} x\right)$ is a first-order approximation to the exact solution for small $\epsilon>0$.

Acknowledgements. The research was partly supported by the National Science Foundation under Grant No. CMS 8903083.

\section{Appendix 1}

Here, we give the explicit expression of $A_{2}$ in (44).

$$
\begin{aligned}
A_{2}=- & \frac{\left(\psi_{0}^{-}\right)^{3}\left(\psi_{0}^{-}+1\right)}{\lambda_{0}\left(C^{-}\right)^{2}\left(1-C^{-} \lambda_{0}\right)}\left[\frac{\left(4-C^{-} \lambda_{0}\right)\left(\psi_{0}^{-}\right)^{2}}{2 C^{-}}+\frac{4 \lambda_{0}\left(\psi_{0}^{-}-\ln \left(1+\psi_{0}^{-}\right)\right)}{1-C^{-} \lambda_{0}}\right] \\
+ & \frac{\left(\psi_{0}^{-}\right)^{3}\left(1-\psi_{0}^{+}\right)}{\lambda_{0}\left(C^{+}\right)^{2}\left(1-C^{+} \lambda_{0}\right)}\left[-\frac{\left(4-C^{+} \lambda_{0}\right)\left(\psi_{0}^{+}\right)^{2}}{2 C^{+}}+\frac{4 \lambda_{0}\left(\psi_{0}^{+}+\ln \left(1-\psi_{0}^{+}\right)\right)}{1-C^{+} \lambda_{0}}\right] \\
+ & \psi_{0}^{+} \psi_{0}^{-}\left\{\left(C^{+}\right)^{-2}\left(1-C^{+} \lambda_{0}\right)^{-3}\right. \\
\times & {\left[\left(\lambda_{0} C^{+} \psi_{0}^{+} \psi_{0}^{-}-\psi_{0}^{+} \psi_{0}^{-}+\psi_{0}^{-}\right)^{2}+(3 / 2)\left(\left(\psi_{0}^{+}-1\right) \psi_{0}^{-}\left(1-C^{+} \lambda_{0}\right)\right)^{2}\right.} \\
+ & \left.2 \psi_{0}^{-}\left(\psi_{0}^{+}-1\right)\left(1-C^{+} \lambda_{0}\right)\left(\lambda_{0} C^{+} \psi_{0}^{+} \psi_{0}^{-}-\psi_{0}^{+} \psi_{0}^{-}+\psi_{0}^{-}\right)\right] \\
- & \left(C^{-}\right)^{-2}\left(1-C^{-} \lambda_{0}\right)^{-3} \\
& \times\left[\left(\lambda_{0} C^{-} \psi_{0}^{+} \psi_{0}^{-}-\psi_{0}^{+} \psi_{0}^{-}-\psi_{0}^{+}\right)^{2}+(3 / 2)\left(\left(\psi_{0}^{-}+1\right) \psi_{0}^{+}\left(1-C^{-} \lambda_{0}\right)\right)^{2}\right. \\
& \left.\left.+2 \psi_{0}^{+}\left(\psi_{0}^{-}+1\right)\left(\lambda_{0} C^{-} \psi_{0}^{+} \psi_{0}^{-}-\psi_{0}^{+} \psi_{0}^{-}-\psi_{0}^{+}\right)\left(1-C^{-} \lambda_{0}\right)\right]\right\} .
\end{aligned}
$$

We note here that $A_{2}$ can be positive, negative, or even zero depending on the values of $C^{+}, C^{+}, \psi_{0}^{-}$, and $\psi_{0}^{+}$. In this paper, we assume $A_{2} \neq 0$.

\section{Appendix 2}

We shall consider the asymptotic behavior of the eigenvalues of (64)-(66) of Lemma

2. Let $\left|\tau_{n}\right|$ be large enough so that $\lambda_{0}^{2}-4\left|\tau_{n}\right| \lambda_{0}\left(C^{ \pm} \lambda_{0}-1\right) C^{ \pm}<0$. If we let

$$
\mu_{n}^{ \pm}=\frac{\left(4\left|\tau_{n}\right| \lambda_{0}\left(C^{ \pm} \lambda_{0}-1\right) C^{ \pm}-\lambda_{0}^{2}\right)^{1 / 2}}{2 \lambda_{0}},
$$

then the solution of (64) satisfying (66) and $e_{n}^{+}=e_{n}^{-}$at $\psi=0$ is

$$
\begin{aligned}
& e_{n}^{+}(\psi)=C(1-\psi)^{-1 / 2} \sin \left(\mu_{n}^{-} \ln \left(1+\psi_{0}^{-}\right)\right) \sin \left(\mu_{n}^{+} \ln \left(\frac{1-\psi}{1-\psi_{0}^{+}}\right)\right), \\
& e_{n}^{-}(\psi)=C(1-\psi)^{-1 / 2} \sin \left(\mu_{n}^{+} \ln \left(1-\psi_{0}^{+}\right)\right) \sin \left(\mu_{n}^{-} \ln \left(\frac{1-\psi}{1+\psi_{0}^{-}}\right)\right),
\end{aligned}
$$

where $C$ is a normalizing factor. From (65), we observe that $\mu_{n}^{ \pm}$must satisfy

$$
\sin \left(\mu_{n}^{-} \ln \left(1+\psi_{0}^{-}\right)\right) \sin \left(\mu_{n}^{+} \ln \left(1-\psi_{0}^{+}\right)\right)
$$




$$
\begin{aligned}
& \times\left[\frac{1}{2 C^{+}\left(1-C^{+} \lambda_{0}\right)}-\frac{1}{2 C^{-}\left(1-C^{-} \lambda_{0}\right)}-\lambda_{0}^{2} \frac{C^{+}-C^{-}}{\left(1-C^{-} \lambda_{0}\right)\left(1-C^{+} \lambda_{0}\right)}\right] \\
- & \frac{\mu_{n}^{+}}{C^{+}\left(C^{+} \lambda_{0}-1\right)} \sin \left(\mu_{n}^{-} \ln \left(1+\psi_{0}^{-}\right)\right) \cos \left(\mu_{n}^{+} \ln \left(1-\psi_{0}^{+}\right)\right) \\
+ & \frac{\mu_{n}^{-}}{C^{-}\left(C^{-} \lambda_{0}-1\right)} \sin \left(\mu_{n}^{+} \ln \left(1-\psi_{0}^{+}\right)\right) \cos \left(\mu_{n}^{-} \ln \left(1+\psi_{0}^{-}\right)\right)=0 .
\end{aligned}
$$

However, from the definition of $\mu_{n}^{ \pm}$, when $\left|\tau_{n}\right| \rightarrow+\infty, \mu_{n}^{ \pm}$go to infinity with order $\left|\tau_{n}\right|^{1 / 2}$. Thus

$$
\begin{aligned}
& C^{-}\left(C^{-} \lambda_{0}-1\right) \sin \left(\mu_{n}^{-} \ln \left(1+\psi_{0}^{-}\right)\right) \cos \left(\mu_{n}^{+} \ln \left(1-\psi_{0}^{+}\right)\right) \\
& \quad-C^{+}\left(C^{+} \lambda_{0}-1\right) \sin \left(\mu_{n}^{+} \ln \left(1-\psi_{0}^{+}\right)\right) \cos \left(\mu_{n}^{-} \ln \left(1+\psi_{0}^{-}\right)\right)=O\left(\frac{1}{\left|\tau_{n}\right|^{1 / 2}}\right) .
\end{aligned}
$$

If we keep the lowest order terms, we have

$$
\begin{aligned}
& C^{-}\left(C^{-} \lambda_{0}-1\right) \sin \left(\left(\frac{\left|\tau_{n}\right|\left(C^{-} \lambda_{0}-1\right) C^{-}}{\lambda_{0}}\right)^{1 / 2} \ln \left(1+\psi_{0}^{-}\right)\right) \\
& \times \cos \left(\left(\frac{\left|\tau_{n}\right|\left(C^{+} \lambda_{0}-1\right) C^{+}}{\lambda_{0}}\right)^{1 / 2} \ln \left(1-\psi_{0}^{+}\right)\right) \\
&-C^{+}\left(C^{+} \lambda_{0}-1\right) \sin \left(\left(\frac{\left|\tau_{n}\right|\left(C^{+} \lambda_{0}-1\right) C^{+}}{\lambda_{0}}\right)^{1 / 2} \ln \left(1-\psi_{0}^{+}\right)\right) \\
& \times \cos \left(\left(\frac{\left|\tau_{n}\right|\left(C^{-} \lambda_{0}-1\right) C^{-}}{\lambda_{0}}\right)^{1 / 2} \ln \left(1+\psi_{0}^{-}\right)\right)=0 .
\end{aligned}
$$

Hence,

$$
\begin{aligned}
\left(C^{-}\left(C^{-} \lambda_{0}-1\right)-C^{+}\left(C^{+} \lambda_{0}-1\right)\right) \sin \left(\left(\frac{\left|\tau_{n}\right|}{\lambda_{0}}\right)^{1 / 2}\right. & \\
& \left.\times\left(\left(\frac{\left(C^{-} \lambda_{0}-1\right) C^{-}}{\lambda_{0}}\right)^{1 / 2} \ln \left(1+\psi_{0}^{-}\right)+\left(\frac{\left(C^{+} \lambda_{0}-1\right) C^{+}}{\lambda_{0}}\right)^{1 / 2} \ln \left(1-\psi_{0}^{+}\right)\right)\right) \\
- & \left(C^{-}\left(C^{-} \lambda_{0}-1\right)+C^{+}\left(C^{+} \lambda_{0}-1\right)\right) \sin \left(\left(\frac{\left|\tau_{n}\right|}{\lambda_{0}}\right)^{1 / 2}\right. \\
& \left.\left(\left(\frac{\left(C^{+} \lambda_{0}-1\right) C^{+}}{\lambda_{0}}\right)^{1 / 2} \ln \left(1-\psi_{0}^{+}\right)-\left(\frac{\left(C^{-} \lambda_{0}-1\right) C^{-}}{\lambda_{0}}\right)^{1 / 2} \ln \left(1+\psi_{0}^{-}\right)\right)\right)=0 .
\end{aligned}
$$

However, the number of intersections of two sine functions is of order $n$. Thus $\left|\tau_{n}\right|^{1 / 2} \sim$ $O(n)$ or $\left|\tau_{n}\right| \sim O\left(n^{2}\right)$. Therefore $\left|\tau_{n}\right| \geq K\left(n^{2}\right)$ for some $K>0$.

Next, we show that $e_{n}(\psi)$ is uniformly bounded as $n \rightarrow+\infty$. Since

$$
\int_{-\psi_{0}^{-}}^{\psi_{0}^{+}} e_{n}^{2}(\psi) d \psi=1
$$

$C$ in $e_{n}(\psi)$ must be chosen by 


$$
\begin{aligned}
C= & \left\{\frac{\sin ^{2}\left(\mu_{n}^{+} \ln \left(1-\psi_{0}^{+}\right)\right)}{4 \mu_{n}^{-}}\left(2 \mu_{n}^{-} \ln \left(1+\psi_{0}^{-}\right)+\sin \left(2 \mu_{n}^{-} \ln \left(1+\psi_{0}^{-}\right)\right)\right)\right. \\
& \left.-\frac{\sin ^{2}\left(\mu_{n}^{-} \ln \left(1+\psi_{0}^{-}\right)\right)}{4 \mu_{n}^{+}}\left(2 \mu_{n}^{+} \ln \left(1-\psi_{0}^{+}\right)+\sin \left(2 \mu_{n}^{+} \ln \left(1-\psi_{0}^{+}\right)\right)\right)\right\}^{-1 / 2} \\
\leq & K\left(\sin ^{2}\left(\mu_{n}^{+} \ln \left(1-\psi_{0}^{+}\right)\right)+\sin ^{2}\left(\mu_{n}^{-} \ln \left(1+\psi_{0}^{-}\right)\right)\right)^{-1 / 2},
\end{aligned}
$$

for $n$ large, where $K$ is independent of $n$. It follows that

$$
\begin{aligned}
\left|e_{n}^{+}(\psi)\right| & \leq C(1-\psi)^{-1 / 2}\left|\sin \left(\mu_{n}^{-} \ln \left(1+\psi_{0}^{-}\right)\right)\right| \\
& \leq K(1-\psi)^{-1 / 2} \leq K_{1}, \\
\left|e_{n}^{-}(\psi)\right| & \leq C(1-\psi)^{-1 / 2}\left|\sin \left(\mu_{n}^{+} \ln \left(1-\psi_{0}^{+}\right)\right)\right| \\
& \leq K(1-\psi)^{-1 / 2} \leq K_{2},
\end{aligned}
$$

where $K_{1}, K_{2}$ are fixed numbers. Thus $e_{n}(\psi)$ is uniformly bounded in $-\psi_{0}^{-} \leq \psi \leq \psi_{0}^{+}$.

\section{References}

1. C. J. Amick and R. E. L. Turner, A global theory of solitary waves in two-fluid systems, Trans. Amer. Math. Soc. 298 (1986), 431-481.

2. _ـ Small internal waves in two-fluid systems, Arch. Rational Mech. Anal. 108 (1989), 111-139.

3. J. Bona and R. L. Sachs, The existence of internal solitary waves in a two-fluid system near the KdV limit, Geophys. Astrophys. Fluid Dynamics 48 (1989), 25-51.

4. L. K. Forbes and S. R. Belward, Atmospheric interfacial waves, Phys. Fluids A 4 (1992), 22222229 .

5. 6 (1994), 3306-3316.

6. D. Gilbarg and N. S. Trudinger, Elliptical Partial Differential Equations of Second Order, Springer-Verlag, New York, 1977.

7. R. H. J. Grimshaw and D. I. Pullin, Extreme interfacial waves, Phys. Fluids 29 (1986), 28022807.

8. D. I. Pullin and R. H. J. Grimshaw, Finite-amplitude solitary waves at the interface between two homogeneous fluids, Phys. Fluids 31 (1988), 3550-3559.

9. Huyun Sha and J.-M. Vanden-Broeck, Solitary waves on water of finite depth with a surface or bottom shear layer, Phys. Fluids 7 (1995), 1048-1055.

10. M. C. Shen, Internal solitary waves in an atmosphere with thermal inversion, J. Atmos. Sci. 24 (1967), 260-266.

11. S. M. Sun and M. C. Shen, Solitary waves in a two-layer fluid with surface tension, SIAM J. Math. Anal. 24 (1993), 866-891.

12. __ Exact theory of generalized solitary waves in a two-layer liquid in the absence of surface tension, J. Math. Anal. Appl. 180 (1993), 245-274.

13. M. Tepper, Pressure jump lines in mid-western United States, Weather Bureau Research Report No. $37,1954$.

14. A. M. Ter-Krikorov, Théorie exact des ondes longues stationnaires dans un liquide hétérogène, J. Mécanique, 3 (1963), 351-375.

15. R. E. L. Turner and J.-M. Vanden-Broeck, Broadening of interfacial solitary waves, Phys. Fluids, 31 (1988), 2486-2490.

16. C. S. Yih, Stratified Fluids, 2nd ed., Academic Press, 1980.

Department of Mathematics, University of Wisconsin, Madison, Wi, 53706, USA

Department of Mathematics, Virginia Polytechnic Institute and State University, BlacksBURG, VA 24061, USA 